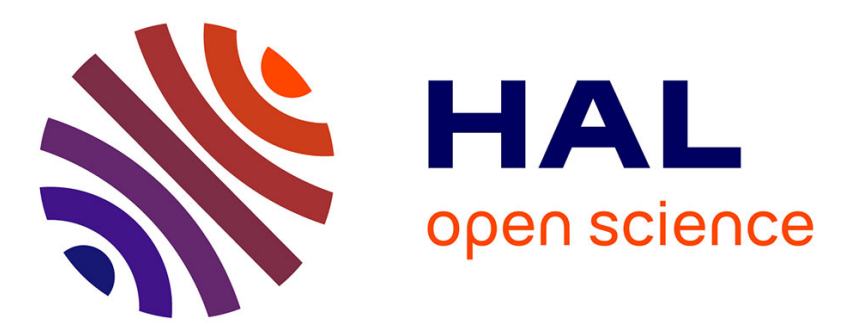

\title{
Structural improvement of zinc oxide films produced by ion beam assisted reactive sputtering
}

D Köhl, M Luysberg, M Wuttig

\section{To cite this version:}

D Köhl, M Luysberg, M Wuttig. Structural improvement of zinc oxide films produced by ion beam assisted reactive sputtering. Journal of Physics D: Applied Physics, 2010, 43 (20), pp.205301. 10.1088/0022-3727/43/20/205301 . hal-00569607

\section{HAL Id: hal-00569607 https://hal.science/hal-00569607}

Submitted on 25 Feb 2011

HAL is a multi-disciplinary open access archive for the deposit and dissemination of scientific research documents, whether they are published or not. The documents may come from teaching and research institutions in France or abroad, or from public or private research centers.
L'archive ouverte pluridisciplinaire HAL, est destinée au dépôt et à la diffusion de documents scientifiques de niveau recherche, publiés ou non, émanant des établissements d'enseignement et de recherche français ou étrangers, des laboratoires publics ou privés. 


\title{
Structural improvement of zinc oxide films produced by ion beam assisted reactive sputtering
}

\author{
D Köhl ${ }^{1}$, M Luysberg $^{2}$ and M Wuttig ${ }^{1,3}$ \\ ${ }^{1}$ I. Institute of Physics (IA), RWTH Aachen University, D-52056 Aachen, Germany \\ ${ }^{2}$ Ernst Ruska-Zentrum für Mikroskopie und Spektroskopie mit Elektronen / IFF, \\ Forschungszentrum Jülich, D-52425 Jülich, Germany
}

\begin{abstract}
Reactively sputtered Zinc Oxide thin films exhibit low crystalline order when deposited on unheated substrates. To improve the structural order, films are usually deposited onto heated substrates at temperatures of about $200-300{ }^{\circ} \mathrm{C}$. Nevertheless, techniques that enable room temperature deposition of $\mathrm{ZnO}$ films with high structural quality would be advantageous. In this work ion bombardment from an auxiliary ion gun during film growth is employed to improve the crystalline quality. $\mathrm{Xe}^{+}$ion bombardment under appropriate conditions leads to the growth of films with high crystalline order. Based on our structural investigations employing x-ray diffraction, atomic force microscopy and transmission electron microscopy, a growth model is proposed which explains the impact of ion bombardment on the structural evolution. We prove that it is especially the nucleation stage of the growth process which is susceptible to this ion bombardment.
\end{abstract}

PACS 81.15.Aa, 81.15.Cd, 81.15.Jj, 68.55.ag, 68.55.jm

\section{Introduction}

Zinc oxide is widely used in many industrial applications. Typical fields cover e.g. surface acoustic wave devices [1], varistors [2], transparent electrodes [3] and thin film transistors [4]. Especially aluminium-doped thin films have increasingly attained focus in recent years as an n-type transparent conducting oxide (TCO) that could potentially replace Indium Tin Oxide (ITO) [5]. Due to the high abundance of both zinc and aluminium, $\mathrm{ZnO}: \mathrm{Al}$ is a low-cost material. Therefore it is the TCO of choice e.g. in the fabrication of thin film solar cells, where it is used as a transparent front electrode [6]. Another important market segment where Zinc Oxide is utilized is the fabrication of Ag based low-emissivity coatings. In this application thin Zinc Oxide buffer layers enable the growth of silver films with improved structural qualities due to the very small epitaxial mismatch between $\mathrm{Ag}$ and $\mathrm{ZnO}$. Optimum performance of these coatings requires the control of both, texture and surface topography of the $\mathrm{ZnO}$ films [7].

Sputtering processes are preferentially applied for the deposition of Zinc Oxide thin films due to the excellent scalability of the process [8] required for coating large area substrates. Sputter-deposited Zinc Oxide thin films exhibit a hexagonal wurtzite structure and predominantly grow with the c-axis oriented perpendicular to the substrate surface. Optimum orientational order would be achieved under perfect thermodynamic equilibrium [9]. However, during thin film growth, the evolution of crystal structure is determined by many different aspects. Besides other deposition parameters, the temperature of the substrate and the growth rate determine the extent of deviation from thermodynamic equilibrium. Therefore, during sputter deposition of Zinc Oxide or aluminium doped Zinc Oxide, the substrates are usually heated to temperatures around $200-300^{\circ} \mathrm{C}$ to yield optimum structural order $[9,10,11]$. This results in increased process costs and also limits the choice of substrates, thereby e.g. hindering the utilization of flexible substrates. These temperatures enable lower nucleation rates as well as defect annihilation, the latter needed to cure the damage caused by the high energetic oxygen ions originating from the cathode [12]. The bombardment of the growing film by these energetic ions is an unavoidable feature of the discharge in reactive sputtering of Zinc

3 Corresponding author: E-mail: wuttig@physik.rwth-aachen.de

I. Institute of Physics (IA), RWTH Aachen University, 52074 Aachen, Germany, Phone: +49 (0)

24180 27155, Fax: +49 (0) 2418022331 
Oxide [13]. From an industrial point of view, it would be desirable to realize growth conditions that allow for high quality films without intentional substrate heating since it adds to product cost, increased energy consumption and system complexity.

In a recent publication [14] we have already demonstrated that tailored ion bombardment from an auxiliary ion gun leads to a striking improvement of film quality and therefore complies with the requirements for large scale fabrication of high quality films on unheated substrates. It is the key objective of the present study to develop a comprehensive understanding of the mechanisms by which the ion bombardment controls structure formation of sputtered Zinc Oxide thin films. The clear identification of these mechanisms finally allows for the development of a growth model which does not only explain the experimental observations but also provides a clear understanding how films with the best possible structure can be produced at low deposition temperature.

\section{Experimental Procedure}

The geometry of the deposition system is shown in figure 1. An ion source (Tectra Gen II) with an additional differential pumping stage [cf 15 for details on the source] has been installed in a sputter deposition system such that simultaneous growth and bombardment of the films by an auxiliary ion gun is possible. Inevitably, in a reactive sputtering process, the sputtering target constitutes a second source of ions. Negatively charged oxygen ions, for example, are created at the racetrack and are accelerated towards the growing film. These ions represent a contribution to the total ion bombardment of the films which is very difficult to control. The bombardment by the oxygen ions may even result in severe structural damage [16]. However, these ions propagate on well-defined trajectories [12]. Therefore, it is possible to use a mechanical shielding device (cf figure 1) to eliminate this detrimental bombardment. With this prerequisite, the auxiliary gun enables a precise control of energetic ion bombardment. In this work, we will refer to this modified sputtering process as Ion Beam Assisted Deposition (IBAD), which is sometimes abbreviated to the term Ion Assisted Deposition (IAD). Typical IBAD processes [17-21] utilize very low pressure deposition techniques such as evaporation or ion beam sputtering. However, in recent years also sputtering discharges have been combined with ion assistance from an auxiliary gun [22]. As this deposition technique is widely used in many industrial large scale applications, a thorough understanding of the impact of ion bombardment during film growth is of scientific and technological relevance.

In the IBAD process, the metallic $\mathrm{Zn}$ target (diameter: $76 \mathrm{~mm}$, purity: $99.99 \%$ ) is sputtered at a constant discharge current of $250 \mathrm{~mA}(\sim 400 \mathrm{~V})$ in a reactive $\mathrm{Ar} / \mathrm{O}_{2}$ atmosphere in oxide mode. A low pressure of $0.2 \mathrm{~Pa}$ is utilized to minimize scattering of the auxiliary ions on their trajectories towards the growing film. The bombarding species (Xe) is fed directly into the plasma cup of the ion source, improving the efficiency of the ionization and the purity of the ion beam. Typical $\mathrm{Xe}^{+}$ion energies are around $180 \mathrm{eV}$, while the ion to atom arrival ratio at the substrate is approximately $2 \%$. This ratio has been defined as the ratio between the $\mathrm{Xe}^{+}$ion flux density at the substrate position and the flux density of $\mathrm{ZnO}$ molecules contributing to the film growth. The ion current has been measured utilizing a flat probe, neglecting the small portion of secondary electrons generated by the collision of ions with the probe. In good approximation, all ions are singly ionized and therefore we can express the ion flux density $N_{\text {Ion }}$ as

$$
N_{\text {Ion }}=\frac{\mathrm{I}_{\text {Ion }}}{\mathrm{F} \times \sqrt{2} \times e},
$$

where $\mathrm{I}_{\mathrm{Ion}}$ is the measured ion current and $e$ is the elementary charge. $F$ is the area of the flat probe and the factor of $\sqrt{2}$ is a geometrical correction taking into account the $45^{\circ}$ tilt angle of the impinging ion beam with respect to the substrate. The flux density of $\mathrm{ZnO}$ molecules $N_{\mathrm{ZnO}}$ can be derived from the deposition rate.

$$
N_{\mathrm{ZnO}}=R \times \frac{\rho_{\mathrm{ZnO}} \times N_{A}}{M_{\mathrm{ZnO}}},
$$

where $R$ is the deposition rate, $N_{A}$ is Avogadro's constant, $\rho_{\mathrm{ZnO}}$ is the mass density and $M_{\mathrm{ZnO}}$ is the molar mass of $\mathrm{ZnO}$ respectively. The relative arrival ratio is then defined by $N_{\text {Ion }} / N_{\mathrm{ZnO}}$.

As a substrate, a silicon (100) wafer with a thin native amorphous oxide layer was used. However, the structural improvements of the $\mathrm{ZnO}$ films discussed in the following also appear if glass substrates are utilized. The substrates have not been intentionally heated. Its temperature has not been measured in 
the present study. However, Joule heating of the substrate by the ion beam is negligible since the maximum energy transfer to the $2 \times 2 \times 0.65 \mathrm{~cm}^{3}$ Si substrate (specific heat capacity: $703 \mathrm{~J} /(\mathrm{kg} \times \mathrm{K})$ ) is approximately $1 \mathrm{~mJ} / \mathrm{s}$ and typical deposition times range from 100 to 1000 seconds.

To study the impact of $\mathrm{Xe}^{+}$ion bombardment, films have been deposited with and without additional ion bombardment, respectively. Bombardment was always employed at the beginning of each deposition (except for the reference films), but was terminated after different lengths of time. The duration of the ion bombardment was varied to determine the effect of ion bombardment in different growth stages. In the following, the notation "IBAD A 1/4" identifies a bombarded film from series A, where bombardment was applied only during the first quarter of growth. The notation "REF A" describes the reference sample that has been grown under identical conditions but without additional bombardment. Table 1 gives an overview of samples and corresponding measurements discussed in this work.

The structural properties are investigated by X-ray diffraction (XRD), utilizing a Philips X'Pert Pro diffractometer with $\mathrm{CuK} \alpha$ radiation, and by transmission electron microscopy (TEM). TEM specimens were prepared by standard techniques, involving mechanical grinding, dimpling and Ar ion milling. Plan view and cross-sectional specimen have been prepared, which allow the investigation of the microstructure parallel and perpendicular to the growth direction, respectively. TEM investigations were performed with a Tecnai F20 operated at $200 \mathrm{kV}$. For investigations of the surface topography, a DI Dimension 3100 Nanoscope atomic force microscope has been utilized. In-situ measurements of the mechanical growth stresses have been performed by a wafer curvature method. This setup has been described in detail elsewhere [23]. Some samples have also been investigated by X-ray reflectometry (XRR) to determine film thickness and mass density.

\section{Experimental Results}

\subsection{X-Ray Diffraction:}

Figure 2 shows diffraction data for $\mathrm{ZnO}$ films from series $\mathrm{A}$. On the left hand side, $\theta-2 \theta$-scans in Bragg-Brentano geometry are shown for five samples differing in the duration of the ion bombardment at the beginning of film deposition. It is apparent that all films grown under initial bombardment have a strongly improved crystalline quality, as the intensity of the (0002) Bragg peak is significantly increased. Rocking Curve scans displayed on the right hand side clearly show that this is due to an improved orientational order of the crystallite c-axis along the substrate normal. The widths of these peaks directly render the quality of the out-of-plane texture of the films. For the bombarded films, the corresponding FWHM is comparable with films deposited on glass at elevated substrate temperatures [cf e.g. 24]. Another striking fact displayed in figure 2 is that apparently bombardment only during the initial growth stage is already sufficient to produce high quality films. Starting from sample IBAD A $1 / 4$, a further prolongation of the bombardment only leads to an angular shift of the (0002) peak $(\theta-2 \theta)$, resulting in a pronounced double peak structure in the intermediate stages $(2 / 4 \& 3 / 4$ of total film grown under bombardment). This shift in the position of the XRD peak is indicative for differences in the film stress.

\subsection{Mechanical Growth Stresses:}

To further elucidate the origin of the shift of the XRD peaks and to relate it to the bombardment during film growth, the mechanical stress of the films has been investigated by means of an in-situ wafer curvature measurement technique [23]. Figure 3 shows the film stress and the instantaneous stress which have both been calculated from the film force and the deposition rate, which was determined after deposition by XRR.

The film stress $\sigma$ is defined as

$$
\sigma=\frac{\text { Film Force per unit length }[\mathrm{Pa} \times \mathrm{m}]}{\text { Film Thickness }[\mathrm{m}]},
$$

while the instantaneous stress $\sigma_{\mathrm{i}}$ is defined by

$$
\sigma_{i}=\frac{\mathrm{d}(\text { Film Force per unit length) }[\mathrm{Pa} \times \mathrm{m}]}{\mathrm{d}(\text { Film Thickness })[\mathrm{m}]},
$$

This means that $\sigma$ represents an average at each stage of film growth whereas $\sigma_{\mathrm{i}}$ may be interpreted e.g. as the differential change of stress induced by each new deposited layer. Thus, sudden changes in 
the stress generating mechanisms should occur as a step in the latter quantity. Figure 3 shows a reference $\mathrm{ZnO}$ sample (no bombardment) in comparison with an IBAD sample that has been bombarded only during the initial half of the growth. From the upper graph it is evident that, while the ion bombardment interacts with the growing film, additional compressive forces act on the film structure as compared to the reference film. When the bombardment is terminated, a very distinct change in the stress generating mechanisms clearly manifests itself in the instantaneous stress (lower graph). The IBAD sample shows an abrupt change from compressive to tensile forces acting on the structure. It is noteworthy that the strength of the tensile contribution seems to significantly exceed the corresponding forces in the reference sample.

\subsection{Surface Topography:}

The evolution of structural differences as a result of the ion bombardment is also evident from the surface topography. To describe the data, in this section we will use the term "grain" to refer to the surface features observed by atomic force microscopy. We are aware of the fact that these features in the AFM images do not necessarily represent crystal grains. However, in later sections experimental evidence will be presented which suggests that the observed surface features might indeed represent crystalline grains.

Since the results shown in figure 2 indicate that the ion bombardment has a major influence already in the early growth stage, extremely thin films have been produced for this investigation. Figure 4 shows the topography of an only $2.2 \mathrm{~nm}$ thin reference sample and a $2.5 \mathrm{~nm}$ thin IBAD sample. The film thicknesses have been deduced from XRR data, which allows determining the average film thickness with angstrom precision.

The images shown in figure 4 have been post-processed with a convolution filter to enhance the contrast of the surface corrugation (cf [14] for the original images). However, all statistical evaluations that will be shown have been applied to the original topography data to avoid an impact of the postprocessing on the analysis. From figure 4 it is evident that there is a striking difference in surface structure, i.e. the IBAD sample shows a more homogenous grain size distribution. This is supported by the RMS roughness that has been calculated to be $0.88 \mathrm{~nm}$ and $0.44 \mathrm{~nm}$ for the reference and the IBAD sample respectively. Differences in topography are clearly visible from image 5 , where a line profile is shown for both films. It is apparent that the surface of the reference sample is governed by a large variation of grain heights and widths while the IBAD sample shows grains with rather equal dimensions in contrast. This interpretation is supported by the surface height distributions shown in figure 6. The graphs have been normalized to ensure that the area under the graph is unity. The centre of mass of the distributions has been adjusted to agree with the thickness determined by XRR. These distributions can be interpreted in the following way: The FWHM is a measure for the roughness $R$ of the surface and corresponds to the average deviation from the mean height $(\mathrm{FWHM}=2 R)$. The shape directly reflects the nature of the topography. The large right hand side tail for the reference sample is due to some grains which are significantly larger and hence higher than the average (cf figure 5). To investigate how the film topography changes with thickness, thicker films were studied as well.

For this purpose, the approximately $120 \mathrm{~nm}$ thick films sputtered for the mechanical stress analysis have been selected for AFM measurements as well. Analogous to figure 4, post-processed images are shown in figure 7.

The structural differences that have already been present for the thin films are even more pronounced for the thick films. There is a marked difference in the lateral structure size and distribution. The line profile shown in figure 8 again displays a large variation in grain heights for the reference sample in striking contrast to the IBAD film. Large differences in lateral sizes are also clearly visible only for the reference, but not for the IBAD sample. The surface height distribution presented in figure 9 clearly highlights the topographical homogeneity that is found for the IBAD sample, whereas the reference film exhibits a rather broad distribution as observed already for the thin reference sample. For the thick films, the width of the distributions corresponds to an RMS roughness of $3.9 \mathrm{~nm}$ for the reference and only $1.37 \mathrm{~nm}$ for the IBAD sample.

As the structure size for the thick films has increased, it was also possible to successfully apply a watershed algorithm on the topography data in order to detect and analyze the grains. Hence, figure 10 shows a statistical analysis of the grains in terms of their equivalent lateral disc diameter. The absolute number of grains with different diameters has been plotted. The smooth lines are a guide to the eyes, while the original curves show some noise. It is evident that the IBAD sample on average exhibits 
much smaller grain diameters (approx. $15 \mathrm{~nm}$ ) than the reference (approx. $24 \mathrm{~nm}$ ) and that there is also a smaller distribution of different grain sizes for the IBAD film compared with the reference.

From a comparison of the surface structure of sample series B and C it is evident that for the reference film a major change in the structure scale occurs upon increasing film thickness. On the other hand, the respective change for the IBAD film is far less pronounced. Apparently, ion bombardment establishes a growth behaviour where the lateral scale of the surface structure exhibits almost no change upon film growth.

\subsection{Microstructure:}

To further unravel the changes in film growth and the influence of the ion bombardment, TEM measurements have been performed on samples REF A and IBAD A 1/4. Figure 11 displays dark field images of these two samples grown without (figs. a, b) and with $\mathrm{Xe}^{+}$bombardment during growth (figs. c, d). In both cases a columnar growth is observed, where columns extend throughout the whole layer. In figure 11a one crystalline column with a conical shape is seen, which grows from a seed of a width of about $10 \mathrm{~nm}$. At the top of the layer the column reaches a width of about $20 \mathrm{~nm}$. In the lower part of the column a Moiré fringe contrast appears, which is caused by the overlap of two slightly misaligned crystalline grains. In the upper part irregular lines appear which are typical for planar faults on the (0002) planes such as stacking faults. In addition to the column, a crystalline grain shows weak contrast at the left hand side of the figure. Obviously, it does not extend in columnar fashion. The corresponding diffraction pattern (figure 11b) reveals that the (0002) reflections of individual $\mathrm{ZnO}$ grains (marked by arrows) extend along an arc of about $\pm 20^{\circ}$ with respect to the growth direction, which is indicative of a textured growth. (Reflections arising from the $\mathrm{Si}$ substrate are marked by circles). For the bombarded sample a similar microstructure is observed, i.e. a columnar growth. In contrast to the non-bombarded sample, for most cases investigated, the diameters of the nuclei are observed to be constant throughout the whole layer with a width of about $15 \mathrm{~nm}$, which confirms the findings from atomic force microscopy (figure 10). This is demonstrated in figure 11c, where two columns with identical in-plane orientations are shown which have a total width of 25-30 nm. Again some few contrast lines appear parallel to the substrate, which are indicative of planar faults. In addition, a fringe contrast with a spacing of $5.4 \pm 0.2 \AA$ is observed, which agrees with the distance of the (0001) planes. The electron diffraction pattern (figure 11d) reveals that the texture is well defined, i.e. the (0002) reflections show only a small deviation of $\pm 5^{\circ}$ with respect to the growth direction.

In plan-view, the diffraction patterns corresponding to the bright field images reveal a striking difference (see figs. $12 \mathrm{a}, \mathrm{c}$ ). The reference sample is characterized by crystallites of almost random distribution around the texture axis, i.e. (0001). This can be deduced from the ring pattern, which reveals closed rings of individual reflections that are perpendicular to the (0001) axis. Only few (0002) reflections are detected, which correspond to crystallites not obeying the textured growth. In contrast, the diffraction pattern of the IBAD sample is dominated by an arc-like intensity distribution along the rings. This implies an almost identical crystallographic orientation of a majority of crystalline grains seen in figure 12c. However, this orientation relationship between neighboring grains does not extend throughout the whole sample, since the arc within the diffraction pattern is found to "rotate", if a different area of the sample is selected. I.a.w. adjacent crystallites show almost the same crystallographic orientation. On larger scales, i.e. $>500 \mathrm{~nm}$, the orientation of the grains is found to vary.

\section{Discussion}

Summarizing the experimental results, $\mathrm{ZnO}$ films grown with $\mathrm{Xe}^{+}$ion beam assisted sputtering exhibit considerably improved structural properties compared to conventionally sputtered films under otherwise similar conditions. This advancement is disclosed in smaller surface corrugations, homogeneous feature sizes on the surface, in particular for thicker films, as well as in an almost identical crystallographic orientation of adjacent crystallites, and a well defined, textured growth, where the c-axis is aligned in growth direction. It should be emphasized that an ion bombardment only during the initial stage of film growth results in the formation of a drastically improved structure and that there is no further improvement for longer durations of ion bombardment. This implies that IBAD induces important structural changes in the nucleation regime. The structural evolution of the following growth stages is solely governed by the improved structure of the nucleation layer. 
In order to understand, in which way IBAD affects the nucleation, the stress within the $\mathrm{ZnO}$ films is inspected closer. Figure 2 reveals that there is a noticeable shift of the (0002) peak towards lower angles as the duration of the bombardment increases. This is caused by an increased distance of the (0002) planes, which is a typical effect of compressive stress in sputtered $\mathrm{ZnO}$ thin films. The pronounced double peak for intermediate ion bombardment durations is indicative for the co-existence of two phases with different lattice spacing. We can immediately understand this feature from the stress behaviour shown in figure 3. There is a significant change in the evolution of instantaneous stress at the time when the auxiliary ion bombardment is stopped. While the ion bombardment is active, the film shows an increased compressive stress as compared to the reference. This is attributed to the implantation of atoms into e.g. interstitial places, resulting in an increased average inter-atomic spacing. Figure 13 displays the ion bombardment induced lattice expansion schematically. In consequence, the increased lattice constant leads to the peak shift in the XRD diffraction pattern. Upon the termination of ion bombardment there is no further implantation and hence the equilibrium interatomic distance is lower. However, now the subsequent layers grow on a textured film with larger inter-atomic spacing (see figure 13). This results in a tensile biaxial film force as detected in the stress measurement. Without implanted interstitials the planar distance in direction of the c-axis is smaller. Hence, the XRD (0002) Bragg peak occurs close to the literature value of about $34.4^{\circ}$ [25]. We can now interpret the double peak feature as contributions from the bottom and top part of the film, where bombardment was active and inactive, respectively.

Since a pronounced structural modification takes already place in the early growth stage, a corresponding change in morphology can be expected for very thin films. This is confirmed by the AFM images of nominally 2.2 and $2.5 \mathrm{~nm}$ thick reference and IBAD samples, respectively (figure 4). Graphs 5-6 provide further confirmation of a considerable change in surface topography upon $\mathrm{Xe}^{+}$ion bombardment in this very early stage of growth. Hence, the structural changes have their origin in the nucleation and coalescence stage of film growth.

In the later stages, grain growth proceeds in columnar fashion. The TEM images (figure 11) clearly show that the reference film exhibits a conical columnar grain growth. This is linked with a competitive, proliferating growth of neighbouring grains, where grains overgrow each other. For IBAD samples the grains appear to grow in a rather parallel columnar fashion. Hence, the competitive behaviour is less pronounced and the columns are therefore assumed to grow with similar speed. These findings and interpretations are supported by the AFM data. While large changes of lateral structure sizes are observed with increasing thickness for the reference sample, the thick IBAD sample shows topographical features in line with the expected parallel columnar growth in non-proliferating fashion.

The most plausible process responsible for the structural changes seen is a forward sputtering mechanism: atomic peening [26,27] (sometimes also referred to as ion peening). This mechanism is characterized by the implantation of near surface atoms into the film by low energy ion bombardment. A number of findings for the IBAD films are consistent with this scenario. First, there is the compressive growth stress that accompanies the ion bombardment, which is evidence for an implantation mechanism. Additional support is the observed densification of the films. From XRR measurements of $20 \mathrm{~nm}$ thick films and subsequent fitting of the data the mass density of standard $\mathrm{ZnO}$ was determined to $5.25 \pm 0.05 \mathrm{~g} / \mathrm{cm}^{3}$, whereas the density of IBAD samples was $5.45 \pm 0.05 \mathrm{~g} / \mathrm{cm}^{3}$ (cf bulk density: $5.61 \mathrm{~g} / \mathrm{cm}^{3}$ ). Second, from experiments with different bombarding species we found that the structure modification strongly depends on the ion species. If oxygen ions are utilized there is no change except for the expected detrimental effects at higher ion energies and fluxes. Argon ions cause a noticeable structural improvement at moderate energies. However, the effect is small compared to a Xenon based IBAD process. This is consistent with the fact that the atomic peening mechanism is governed by the effective momentum (rather than energy) transfer to the film $[28,29,30]$. Due to its small mass, a reflecting oxygen ion transfers only a small fraction of its momentum to the growing film. There is a better match between the masses of Argon and Zinc Oxide, which explains the small positive effects that have been found. Xenon however is assumed to transfer a large fraction of momentum to the growing film in a multi-collision cascade due to its large mass. This allows for a significantly increased momentum transfer (compared to Argon ions) at moderate fluxes and small energies. It is also important to mention that the dynamic nature of the IBAD 
sputtering process seems to play an important role in structure modification by ions. Experiments have also been performed where film growth and ion bombardment have been performed sequentially. Very thin layers (nominally $<1 \mathrm{~nm}$ ) have been deposited and subsequently bombarded for different times. The procedure has been repeated 8 times before a thick layer has been deposited to yield a total film thickness of approximately $60 \mathrm{~nm}$. None of the resulting films shows any structural modification due to an improved nucleation layer, which is in strong contrast to the films grown under simultaneous ion bombardment. For standard sputtered $\mathrm{ZnO}$ samples it is often reported [31,32] that in the early growth stage the films are rather non-textured, i.e. they consist of small grains with very different crystallographic orientations. It is also reported that in crystal growth processes the (0002) face exhibits a much faster growth speed than the other faces [33]. In [32] it was argued that this might also apply to the growth of sputtered films. Therein, a corresponding growth model was suggested and it was also argued that the negative oxygen ions have a significant albeit detrimental influence on film growth. The orientational disorder at the early growth stage in combination with different growth speeds of the crystallographic orientations would explain why there is competitive growth that finally results in a (0002) texture for the standard samples. The connection between the atomic peening mechanism and texture control presumably originates from the fact that the (0002) planes are the most densely packed planes. Densification of the structure by atomic peening therefore supports a preferred orientation with the (0002) planes parallel to the substrate. Hence, ion bombardment influences the film texture already in the nucleation stage. The fact that the ion bombardment leads to a well defined orientational order already in the early growth stage is supported by an XRD scan of a very thin film, which shows a pronounced (0002) peak (figure 14).

The grain height has been estimated with the Scherrer equation:

$$
D_{G S}=\frac{K \times \lambda}{B \times \cos \theta},
$$

where $D_{G S}$ is the vertical grain size, $\lambda$ is the wavelength of the radiation used, $B$ is the FWHM of the Bragg peak in radians and $\theta$ is the diffraction angle. The constant $K$ depends on the method used to determine $B$ and typically varies between 0.89 and 1 . It has been set to 0.9 here. The fact that there is a detectable Bragg peak intensity at all and that the grain height approximately matches the thickness of this very thin film proves that there is a highly ordered crystalline structure already present at this early stage.

The exclusive formation of highly oriented grains already in the nucleation phase eliminates a competitive growth mode from the very beginning. This also explains why no further ion bombardment is necessary after the initial growth stage.

From the presented data and the foregoing discussion a detailed model of the growth of $\mathrm{ZnO}$ thin films is now proposed, which demonstrates which role the $\mathrm{Xe}^{+}$ion bombardment plays in the IBAD process (figure 15). A distinction between IBAD samples and the standard reference films is already evident after the initial nucleation regime (1). For the reference sample there is a rather random distribution of crystallite orientations (1a). Consequently, surface roughness and lateral structure size distribution are first indications for the onset of competitive growth. Some grains already exhibit larger surfaces and start to overgrow others due to different growth speeds along the various orientations. This behaviour further develops as the film grows thicker (2a). At some stage (3a) the first grains are fully overgrown mainly by crystallites growing in (0001) direction as a result of the proliferating growth. This leads to a broad distribution in the alignment of the (0002) crystal planes which is responsible for the poor crystalline quality that is observed in the XRD measurement. For the IBAD sample atomic peening leads to a preferred orientation already during the nucleation regime (1b). Hence, there is a noncompetitive growth already at this early growth stage which continues in subsequent stages $(2 b \& 3 b)$. Even when the ion bombardment is already interrupted when approximately $3 \mathrm{~nm}$ of the film have been grown (not shown here), thick films with exceptionally high crystal quality are obtained. This demonstrates that stages (2) and (3) in terms of crystal quality are solely governed by the ultra-thin nucleation layer produced in stage (1). Hence, it is especially the nucleation regime that is susceptible to ion bombardment. This observation is important for applications where a perfection of film structure is required. 
Due to the technological importance of texture formation in thin films, a number of studies have focussed on this topic. We will hence relate the present findings with previous studies of texture formation in $\mathrm{ZnO}$ films employing different deposition techniques. Particularly noteworthy is a work from Claeyssens and co-workers [34] where $\mathrm{ZnO}$ films have been deposited using an ArF-PLD process. Substrate heating for different durations revealed that for the fabrication of highly textured films it is sufficient to apply the heating only during the early stage of growth. This finding is in line with our experiments which also demonstrate the importance of the nucleation stage. Both studies provide compelling evidence that the texture of the films is solely determined by the conditions that govern the nucleation regime. Apparently this statement even holds for different thin film fabrication techniques.

There are also studies that link mechanical stresses to texture evolution. Materials that exhibit a large anisotropy in their elastic properties tend to grow in a preferred orientation under the influence of mechanical stresses [35]. However, the anisotropy in the Zinc Oxide compliance tensor [36] is by far less pronounced than for the films reported on in [35]. Therefore the growth stresses generated by the $\mathrm{Xe}^{+}$ion bombardment are presumably not the main reason for texture formation in thin films of Zinc Oxide. We also exclude any processes based on preferential ion sputtering or damage of specific grain orientations due to channelling [37,38] since the $\mathrm{Xe}^{+}$ion energies utilized are much smaller than typical energies required for this effect. Also, the inclination of the ion beam of approximately $45^{\circ}$ would not result in the (0002) texture as the corresponding channelling direction is in the substrate plane $\left(90^{\circ}\right)$.

\section{Conclusion}

$\mathrm{Xe}^{+}$ion bombardment during the growth of reactively sputtered Zinc Oxide films was shown to lead to a distinct improvement in the films' crystalline quality. The structural changes already occur in the nucleation stage, which governs the texture evolution of the whole film. This finding appears to be a fundamental property of Zinc Oxide thin films. The impact of ion bombardment can be explained in terms of an atomic peening mechanism. Hence a deposition process has been developed that yields high quality Zinc Oxide films on unheated substrates.

\section{Acknowledgements}

Financial support by the Deutsche Forschungsgemeinschaft (Wu 243/13) is gratefully acknowledged.

\section{References}

[1] Yoshino Y, Makino T, Katayama Y and Hata T 2000 Vacuum 59 538-545

[2] Gupta T K 1990 J. Am. Ceram. Soc. 73(7) 1817-1840

[3] Szyszka B 1999 Thin Solid Films 351 164-169

[4] Fortunato E M C, Barquinha P M C, Pimentel A C M B G, Gonçalves A M F, Marques A J S, Martins R F P and Pereira L M N 2004 Appl. Phys. Lett. 85(13) 2541-2543

[5] Ellmer K, Klein A and Rech B 2008 Springer Series in Materials Science 104: Transparent Conductive Zinc Oxide ed R Hull, R M Osgood Jr, J Parisi and H Warlimont (Heidelberg: Springer)

[6] Müller J, Schöpe G, Kluth O, Rech B, Sittinger V, Szyszka B, Geyer R, Lechner P, Schade H, Ruske M, Dittmar G, Bochem H P 2003 Thin Solid Films 442 158-162

[7] Arbab M 2001 Thin Solid Films 381 15-21

[8] Müller J, Schöpe G, Kluth O, Rech B, Ruske M, Trube J, Szyszka B, Jiang X and Bräuer G 2001 Thin Solid Films 392 327-333

[9] Park K C, Ma D Y and Kim K H 1997 Thin Solid Films 305 201-209

[10] Agashe C, Kluth O, Hüpkes J, Zastrow U, Rech B and Wuttig M 2004 J. Appl. Phys. 95(4) 1911

[11] Kluth O, Rech B, Houben L, Wieder S, Schöpe G, Beneking C, Wagner H, Löffl A and Schock H W 1999 Thin Solid Films 351 247-253

[12] Severin D, Kappertz O, Nyberg T, Berg S and Wuttig M 2007 Thin Solid Films 515(7-8) 3554-3558

[13] Tominaga K, Murayama T, Sato Y and Mori I 1999 Thin Solid Films 343-344 81-84

[14] Köhl D, Luysberg M and Wuttig M 2009 Phys. Status Solidi RRL 3(7-8) 236-238 
[15] Tectra GmbH, www.tectra.de

[16] Kappertz O, Drese R, Ngaruiya J M, Wuttig M 2005 Thin Solid Films 484 64-67

[17] Colligon J S 2004 Phil. Trans. R. Soc. Lond. A 362 103-116

[18] Ebert J 1990 Surface and Coatings Technology 43/44 950-962

[19] Mattox D M 1989 J. Vac. Sci. Technol. A 7(3) 1105-1114

[20] Colligon J S 1995 J. Vac. Sci. Technol. A 13(3) 1649-1657

[21] Martin P J 1986 Journal of Materials Science 21 1-25

[22] Deniz D, Harper J M E 2008 J. Appl. Phys. 104063519

[23] Drese R J and Wuttig M 2005 J. Appl. Phys. 98073514

[25] Powder Diffraction Files, Joint Committee on Powder Diffraction Standards, JCPDS, Card 800075, 1999.

[26] d'Heurle F M and Harper J M E 1989 Thin Solid Films 171 81-92

[27] Davis C A 1993 Thin Solid Films 226 30-34

[28] Windischmann H 1992 Crit. Rev. in Solid State and Mat. Sci. 17(6) 547-596, pp. 583

[31] Yoshino Y, Inoue K, Takeuchi M and Ohwada K 1998 Vacuum 51(4) 501-507

[32] Petrov I, Orlinov V and Misiuk A 1984 Thin Solid Films 124 55-67

[33] Hu J Q, Quan Li, Wong N B, Lee C S and Lee S T 2002 Chem. Mater. 14 1216-1219

[34] Claeyssens F, Freeman C L, Allan N L, Sun Y, Ashfold M N R and Harding J H 2005 J. Mater. Chem. 15 139-148

[35] McKenzie D R 1993 J. Vac. Sci. Technol. B 11(5) 1928-1935

[36] Özgür Ü, Alivov Y I, Liu C, Teke A, Reshchikov M A, Doğan S, Avrutin V, Cho S J, and Morkoç H 2005 J. Appl. Phys. 98041301

[37] Dobrev D 1982 Thin Solid Films 92 41-53

[38] Dong L, Srolovitz D J 1999 Appl. Phys. Lett. 75(4) 584-586

[24] Yoshino Y, Inoue K, Takeuchi M and Ohwada K 1998 Vacuum 51(4) 601-607

[29] Hoffman D W and Gaerttner M R 1980 J. Vac. Sci Technol. 17(1) 425-428

[30] Kester D J and Messier R 1992 J. Appl. Phys. 72(2) 504-513

\section{List of figures:}
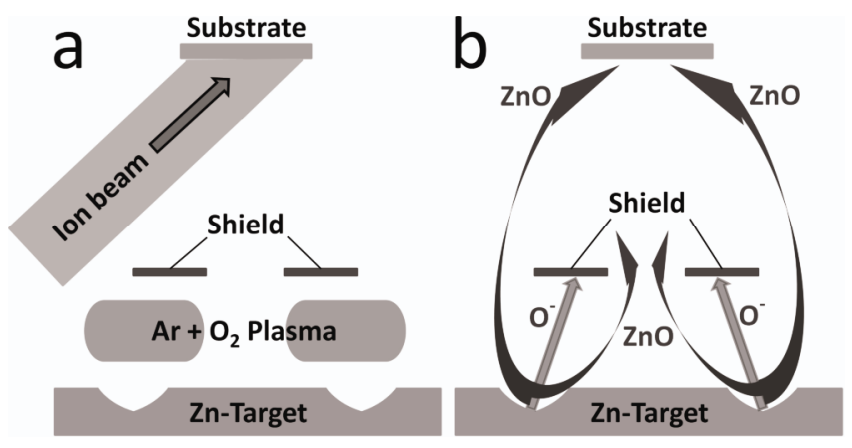

Figure 1. Geometry of the IBAD process: A zinc target is sputtered in a reactive $\mathrm{Ar} / \mathrm{O}_{2}$ atmosphere to yield stoichiometric Zinc Oxide films. a) An auxiliary ion gun is installed to simultaneously enable a well controlled ion bombardment at an inclination angle of approx. $45^{\circ}$. b) A mechanical shield is utilized to avoid the undesirable bombardment of the growing film with oxygen ions originating from the oxidized target surface. While these ions are blocked there is still a partially diffusive $\mathrm{ZnO}$ particle flux to the substrate. 

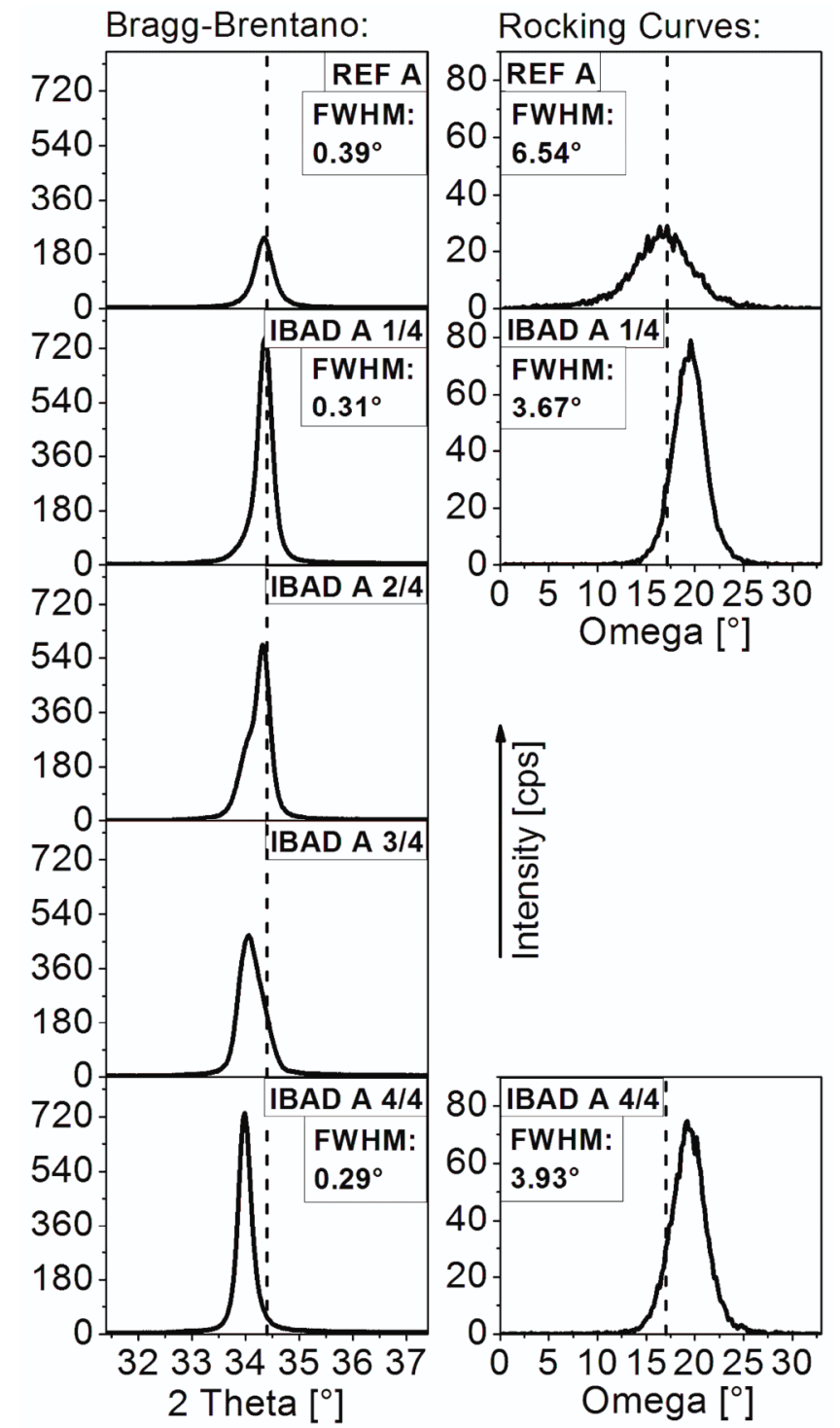

Figure 2. Left: XRD scans in $\theta-2 \theta$ geometry. Right: Corresponding Rocking Curve measurements. The two graphs at the top show an un-bombarded reference sample, which is characterized by a weak intensity of the wurtzite (0002) peak and a broad distribution of crystallite orientations as evident from the rocking curve. Despite identical film thicknesses, all films grown with initial auxiliary ion bombardment show strongly increased peak intensities. This fact is most pronounced for films grown under short (1/4) or long (4/4) duration of the bombardment. The structural enhancement is also evident from the corresponding rocking curves that give evidence for a strongly improved orientational order. The dashed lines represent the $2 \theta$ literature value of $34.4^{\circ}$ for the $\theta-2 \theta$ scans. For the Rocking Curves it represents the position of $\theta$. 


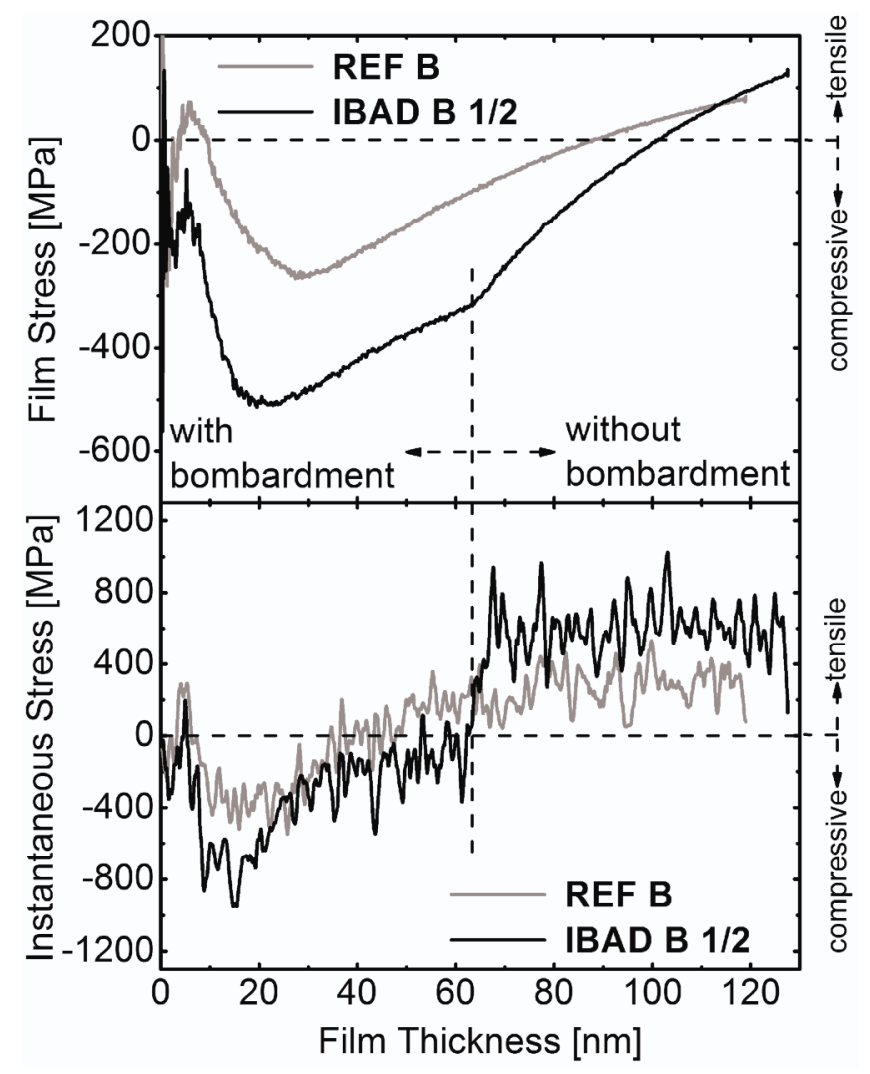

Figure 3. Upper graph: In-situ measurement of the mechanical stress for a reference sample (no $\mathrm{Xe}^{+}-$ ion bombardment) and an IBAD sample that has been bombarded during the first half of the growth time. It is evident that the ion bombardment causes an additional amount of compressive stress. Lower graph: The instantaneous stress represents the differential change in film stress and is therefore sensitive to abrupt changes in the stress generating mechanisms. The interaction of the ion beam with the growing film is apparent in this graph, where a sudden change in the stress generating mechanisms manifests itself in a jump from compressive to tensile forces when the auxiliary ion bombardment is terminated. 


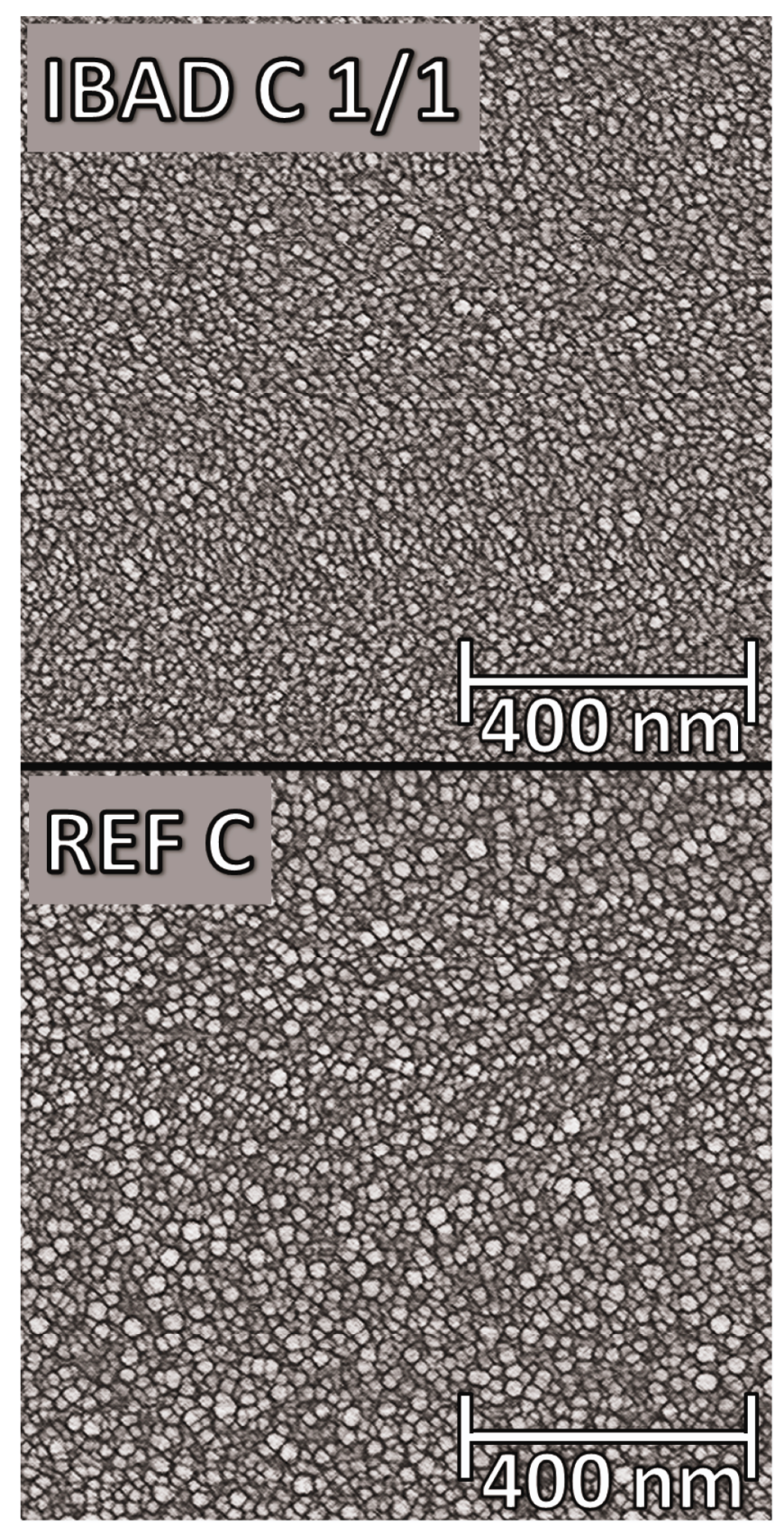

Figure 4. Surface structure of a $2.2 \mathrm{~nm}$ thick standard $\mathrm{ZnO}$ sample and a $2.5 \mathrm{~nm}$ thick IBAD sample. Images have been post-processed (for this figure only) to highlight the surface corrugation.

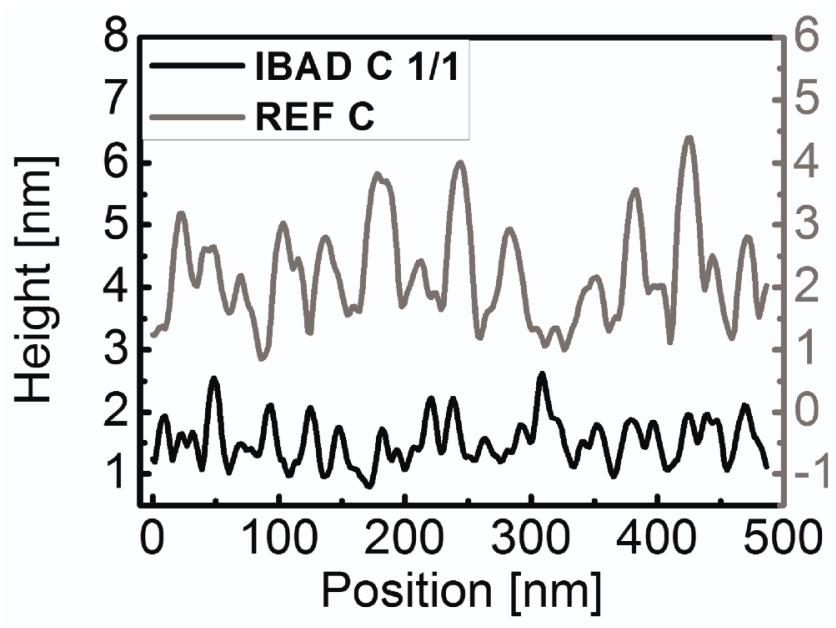


Figure 5. Cross-sectional height profile of the surfaces shown in figure 4 . The IBAD sample shows a smaller distribution of grain heights than the reference.

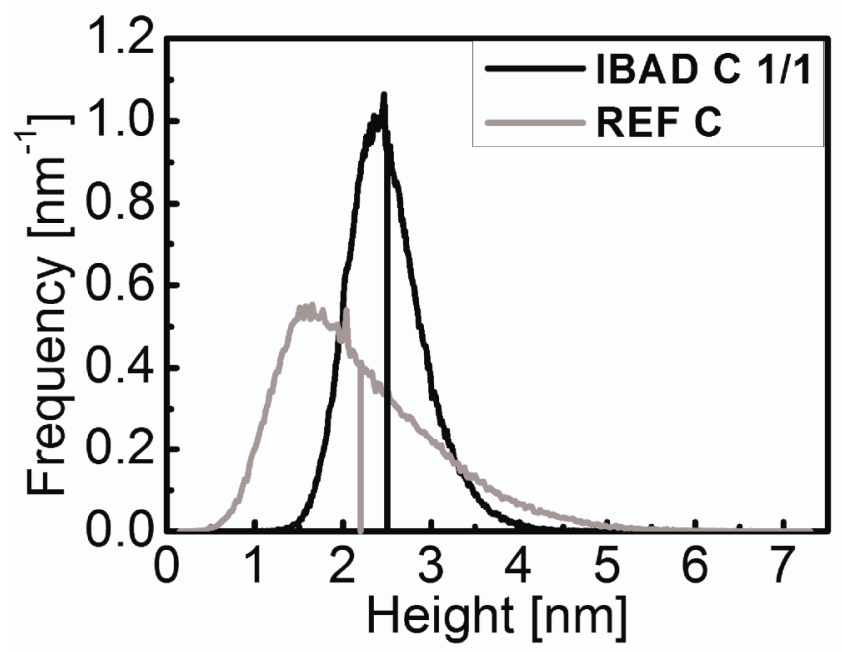

Figure 6. Height distribution of the thin films shown in figure 4. The centre of the distributions (vertical lines) is adjusted to represent the average film thickness determined by XRR. 


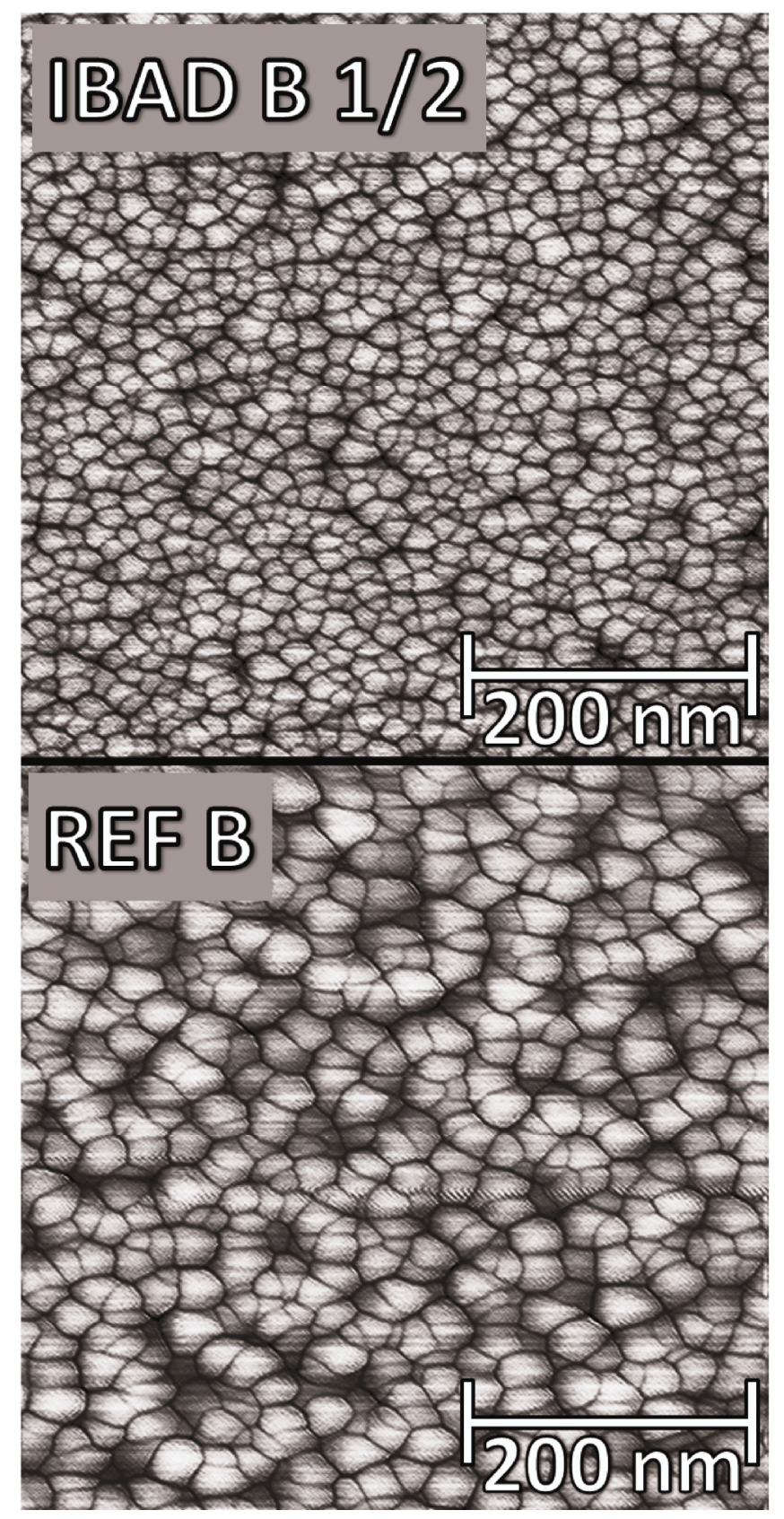

Figure 7. Surface structure of a $127 \mathrm{~nm}$ thick IBAD sample and a $119 \mathrm{~nm}$ thick standard $\mathrm{ZnO}$ sample (reference). The IBAD sample shows a smoother surface and a narrower distribution of grains sizes. The images have been post-processed (for this figure only) to highlight structural features. 


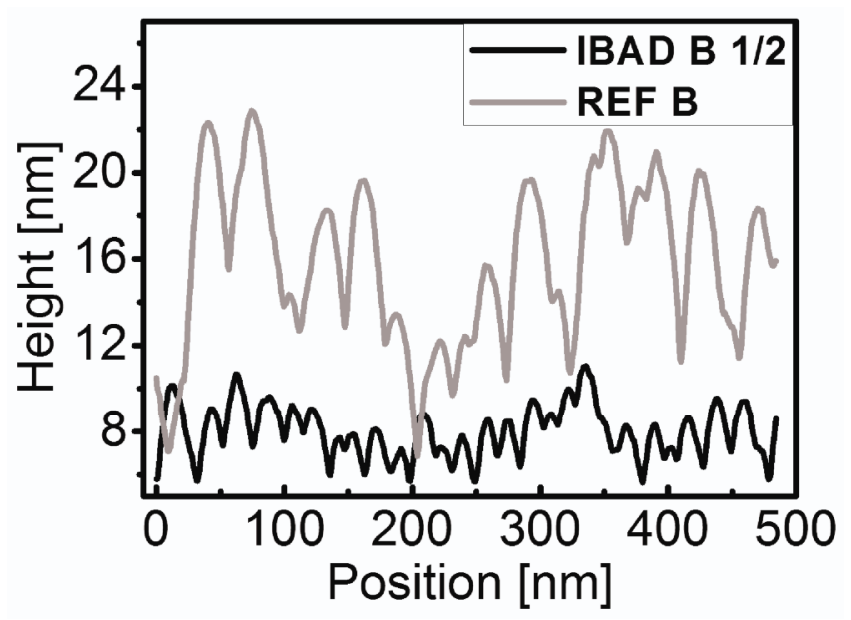

Figure 8. Cross-sectional height profile of the surfaces shown in figure 7. In contrast to the IBAD sample, the reference shows a larger lateral structure size and a stronger variation in the heights of individual grains.

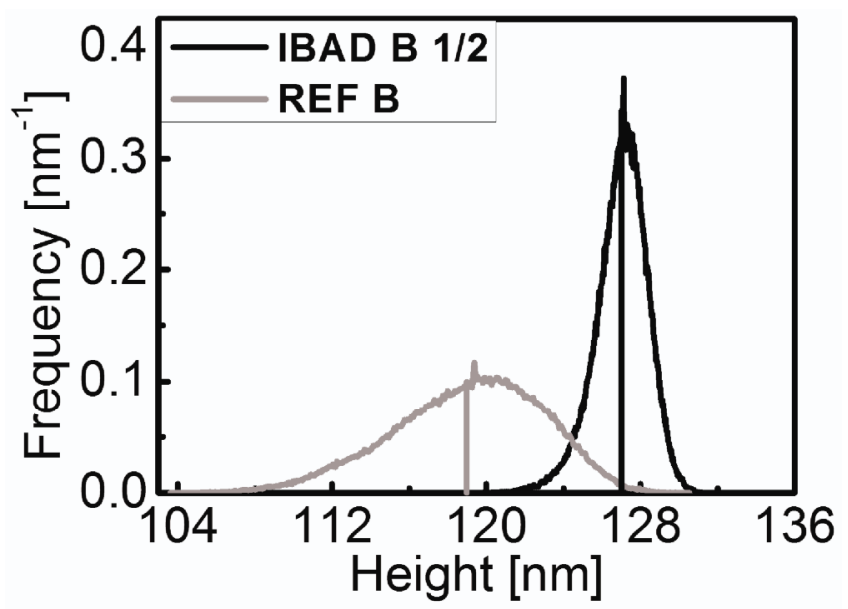

Figure 9. Height distribution of the surfaces shown in figure 7. As for the thin films (figure 6), the centre line of the distributions corresponds to the XRR thickness. The distribution of the IBAD sample is very narrow compared to the standard $\mathrm{ZnO}$ sample.

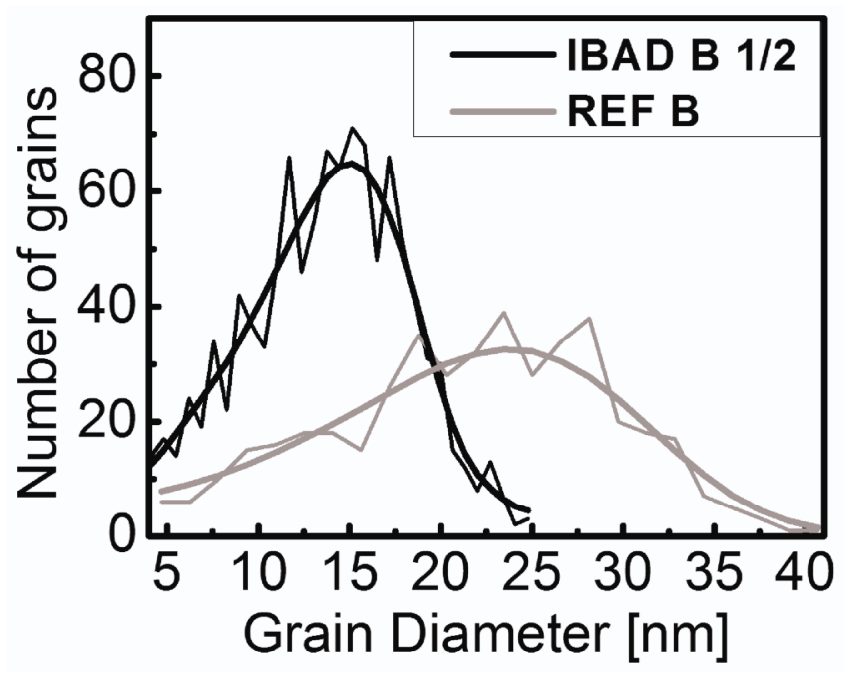

Figure 10. The surfaces of the films discussed in figures 7-9 have been analyzed with a watershed algorithm to detect grains and measure their size. The graph shows the distribution of the equivalent 
disc diameter of the grains. The IBAD sample shows a lower mean grain diameter (lateral structure size) and a narrower distribution.

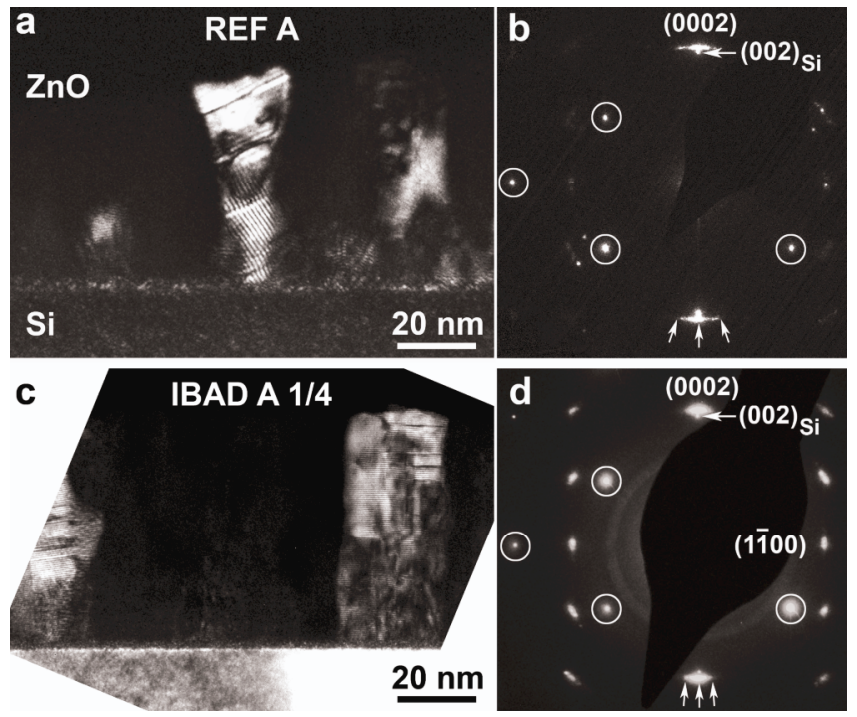

Figure 11. TEM images of a standard $\mathrm{ZnO}$ sample $(\mathrm{a}, \mathrm{b})$ and an IBAD sample $(\mathrm{c}, \mathrm{d})$ respectively. The images on the left $(\mathrm{a}, \mathrm{c})$ show dark field images where single columnar grains are visible. The corresponding diffraction patterns are shown on the right $(b, d)$. Circles mark reflections from the $\mathrm{Si}$ substrate. Some of the Si reflections almost coincide with a (0002) $\mathrm{ZnO}$ reflection and are therefore marked with a single arrow at the top of the images. The arrows in the lower half of figures $(b, d)$ point to the (0002) reflections of $\mathrm{ZnO}$.

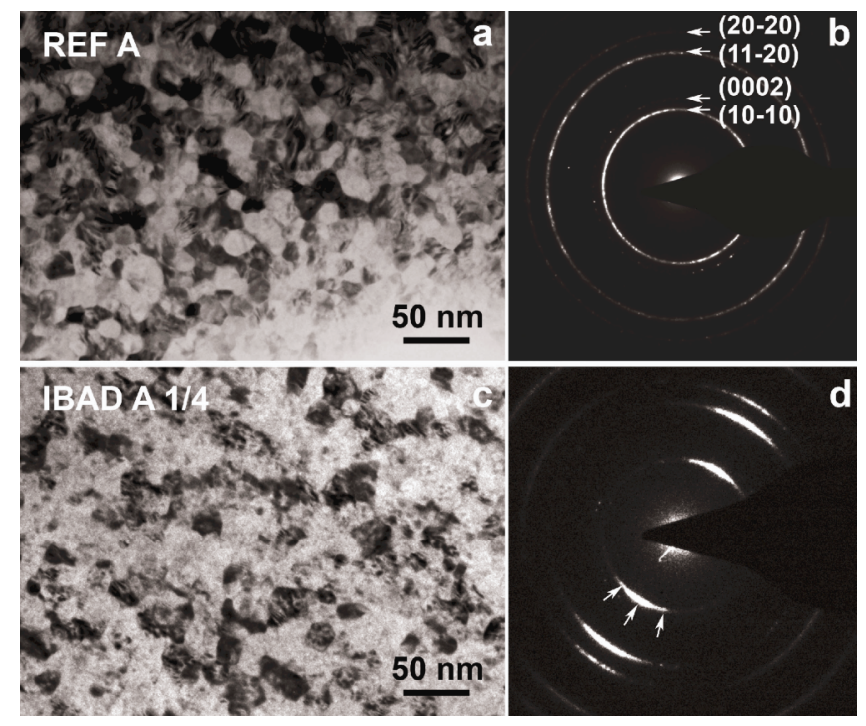

Figure 12. Plan-view images of samples REF A and IBAD A 1/4. The bright field images (a, c) show almost identical grain sizes at the surface. The crystallites of sample REF A are randomly distributed around the texture axis, evident from the ring pattern (b). The IBAD sample reveals some degree of inplane texture, evident from the intensity distribution along an arc in the ring pattern (d). However, there is no long range order, as the arc segment "rotates" when a different area of the sample is scanned. 


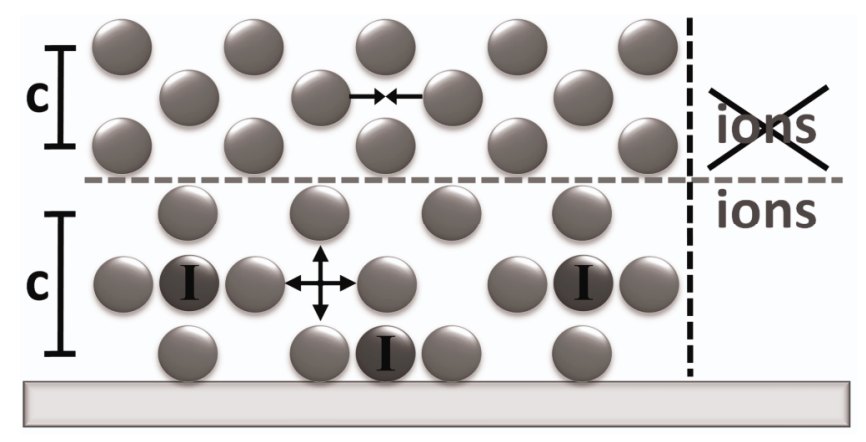

Figure 13. Ion bombardment leads e.g. to interstitials (atoms marked with an "I") that expand the lattice by exerting forces in all directions in space. If bombardment is interrupted, epitaxial growth on the expanded layer leads to tensile biaxial forces because equilibrium conditions have changed. There also occurs a change in the distance between the (0002) planes.

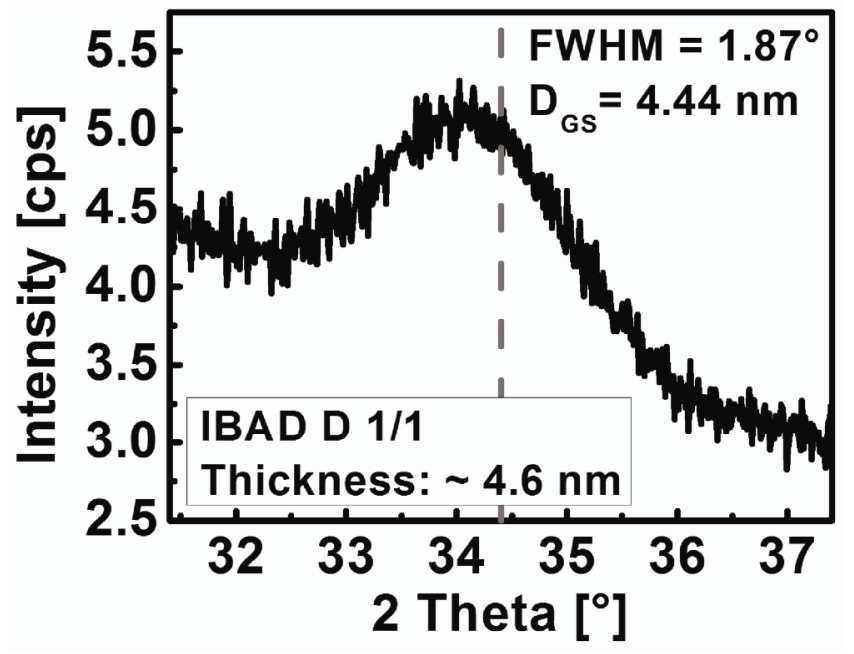

Figure 14. Bragg-Brentano XRD scan of a $4.6 \mathrm{~nm}$ thick IBAD $\mathrm{ZnO}$ sample. A pronounced (0002) Bragg peak with a FWHM of $1.87^{\circ}$ is observed, which corresponds to a grain height of approximately $4.4 \mathrm{~nm}$.

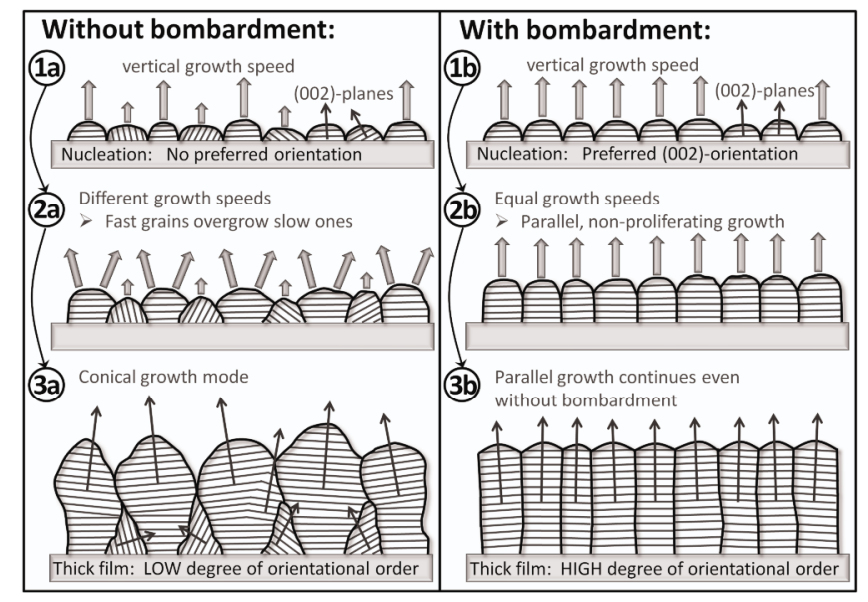

Figure 15. Growth model for standard $\mathrm{ZnO}$ (without auxiliary ion bombardment) and IBAD $\mathrm{ZnO}$ (with auxiliary bombardment). Sketches (1) to (3) represent different growth stages of the films, starting with the nucleation regime (1). The section lining indicates the orientation of the (0002) planes in each crystallite. There is no preferred orientation of grains during nucleation of the standard sample (1a). Different growth speeds (indicated by the vertical arrows) of the different crystal facets result in a competitive growth where the (0002) oriented grains overgrow neighbouring grains with different orientations (2a). This leads to conical grains and poor order of crystallite orientation (3a). In case of 
the bombarded sample, a highly (0002) oriented film is established by an atomic peening mechanism already in the nucleation regime (1b). Hence, competitive growth is avoided (2b) and the resulting structure consists of parallel columnar grains in combination with a high degree of orientational order (3b).

\begin{tabular}{lccc}
\hline Sample & Thickness $(\mathrm{nm})$ & Ion bombardment & Measurement \\
\hline REF A & 82 & none & XRD, TEM \\
\hline IBAD A $1 / 4$ & 81 & stopped after $20 \mathrm{~nm}$ & TEM \\
\hline IBAD A 2/4 & 81 & stopped after $40 \mathrm{~nm}$ & XRD \\
\hline IBAD A 3/4 & 79 & stopped after $60 \mathrm{~nm}$ & XRD \\
\hline IBAD A 4/4 & 80 & yes & XRD \\
\hline REF B & 119 & none & AFM \\
\hline IBAD B $1 / 2$ & 127 & stopped after 63 nm & AFM \\
\hline REF C & 2.2 & none & AFM \\
\hline IBAD C $1 / 1$ & 2.5 & yes & AFM \\
\hline IBAD D $1 / 1$ & 4.6 & yes & XRD \\
\hline
\end{tabular}

Table 1. List of samples and corresponding measurements explicitly discussed in this work. 

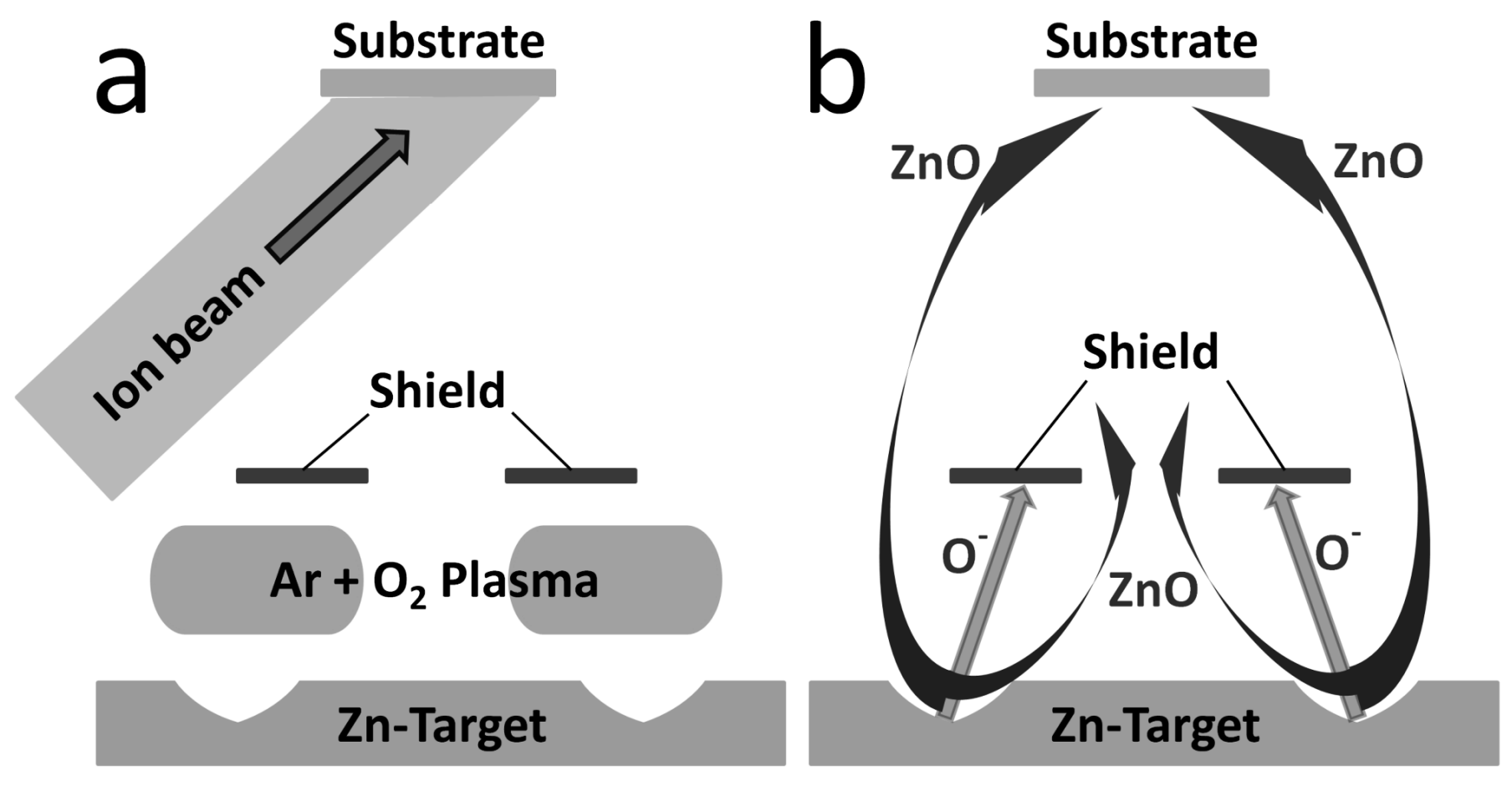

Figure 1 (Figure_1.tif) 


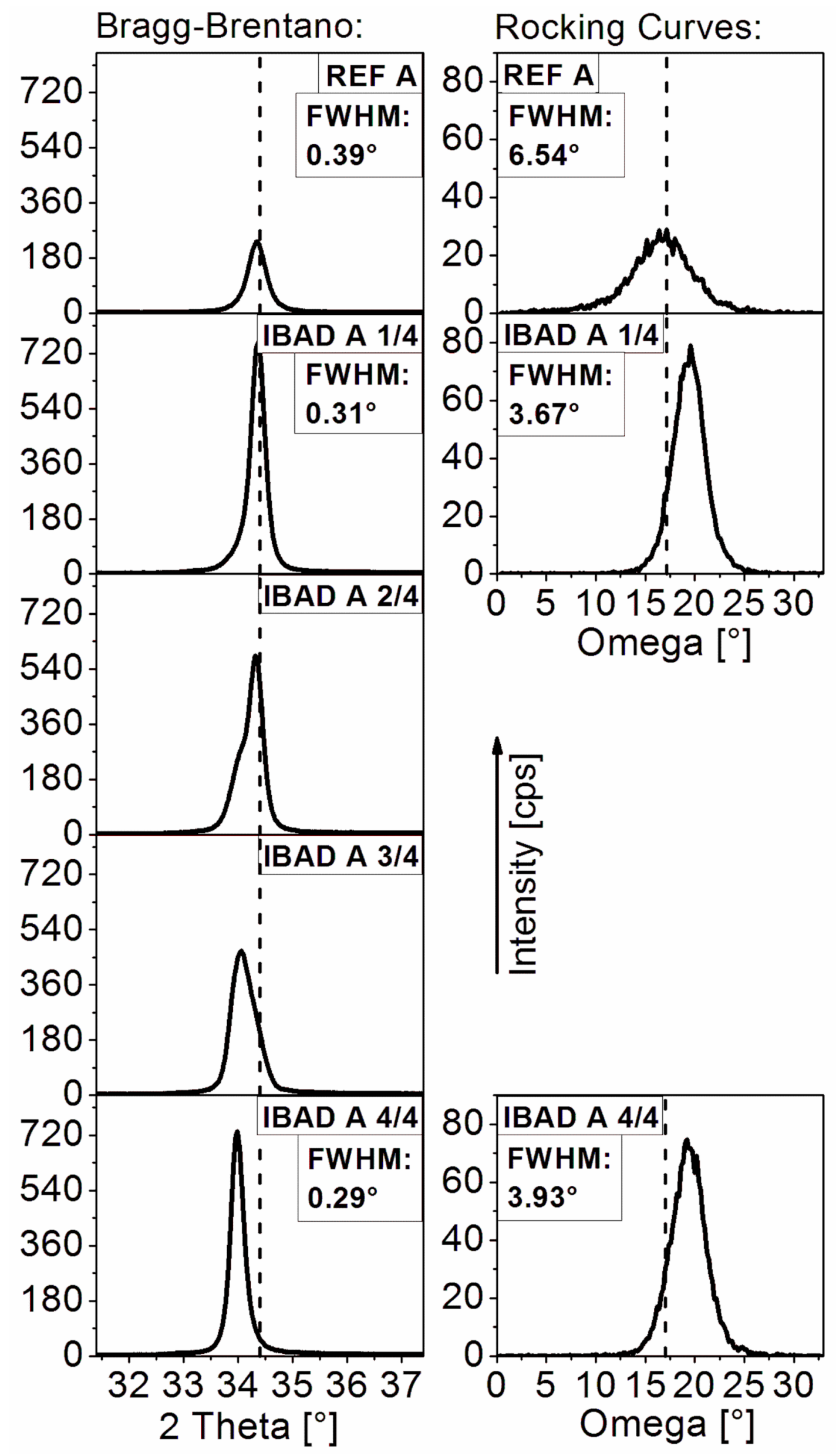

Figure 2 (Figure_2.tif) 


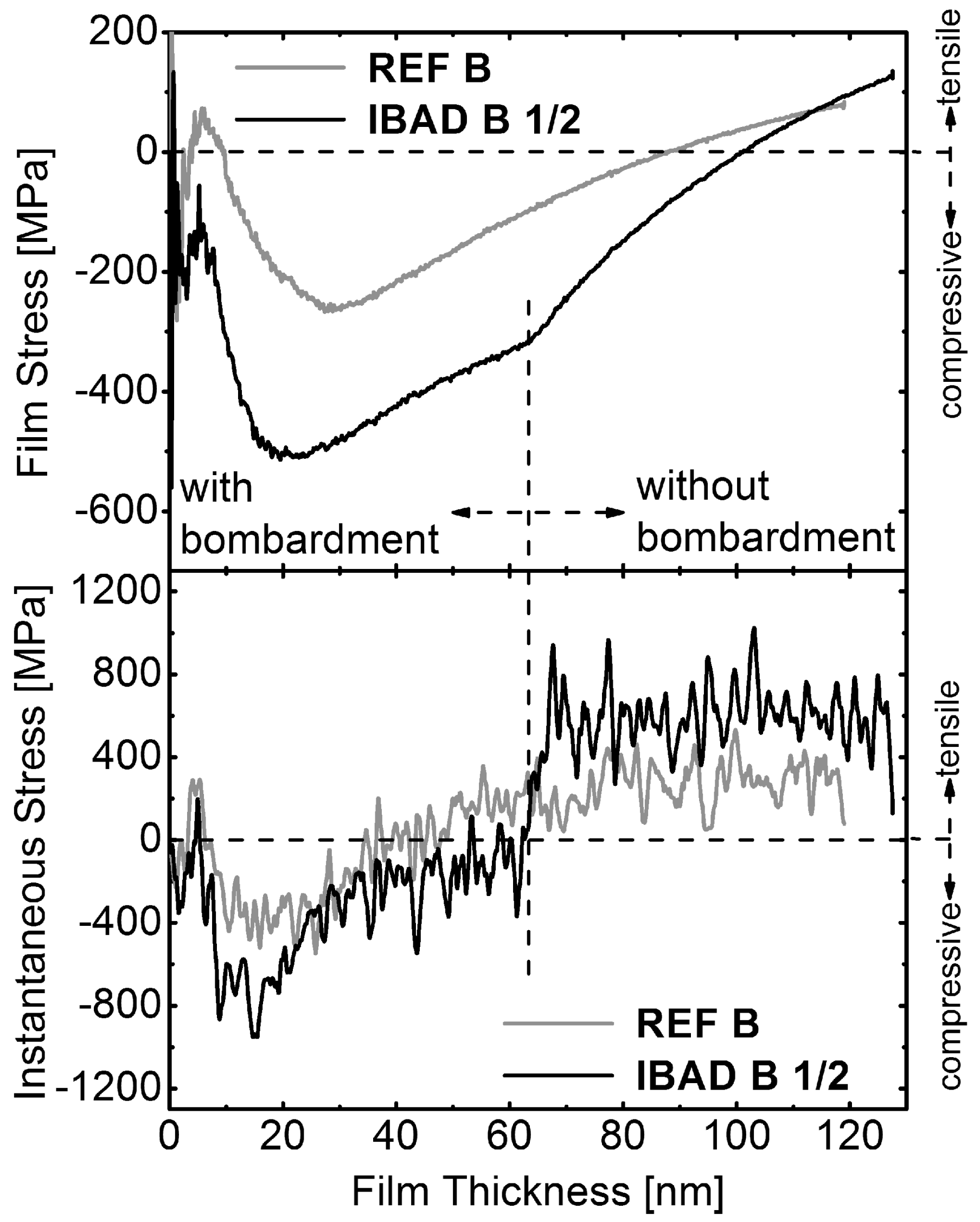

Figure 3 (Figure_3.tif) 


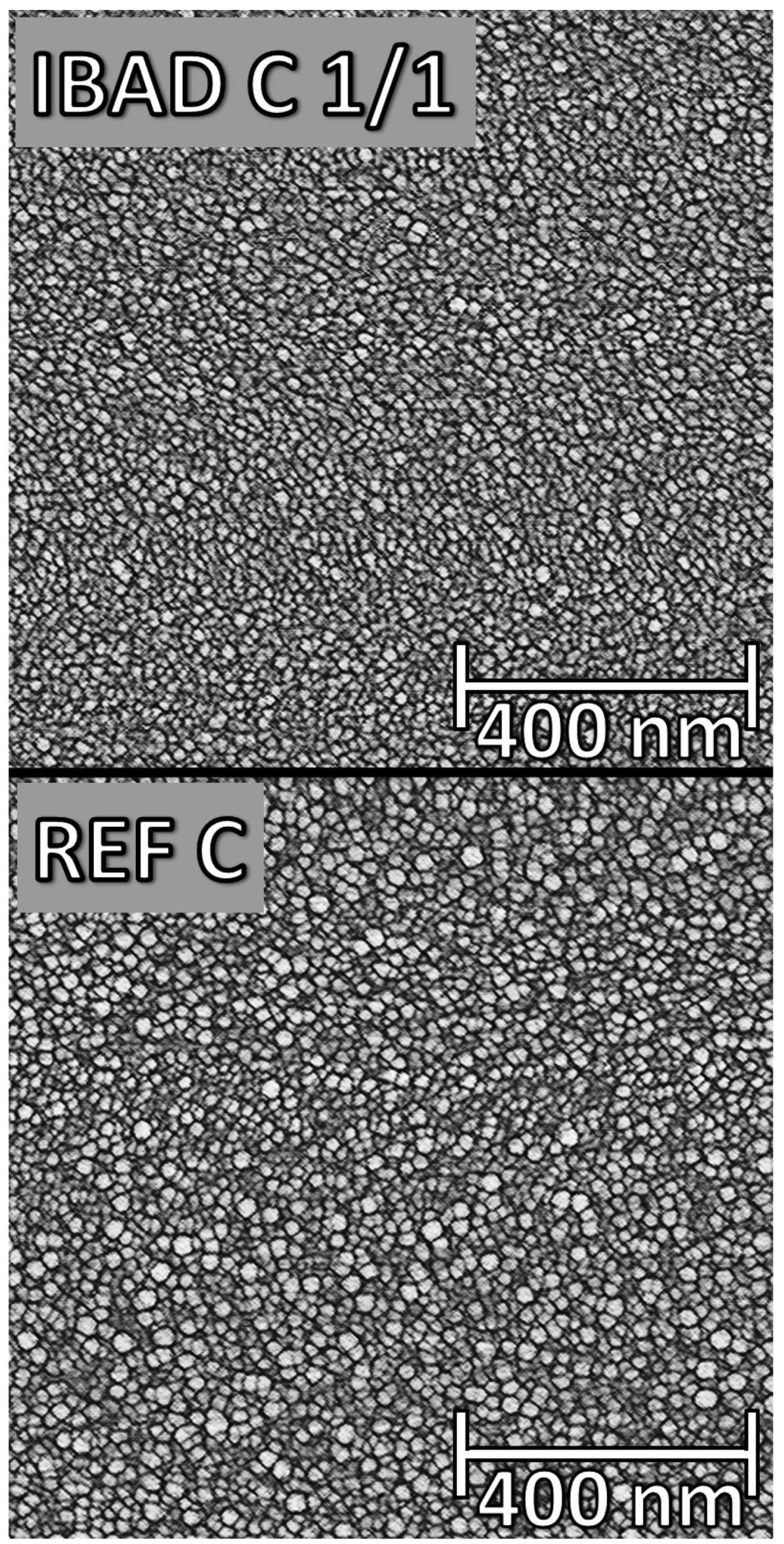

Figure 4 (Figure_4.tif) 


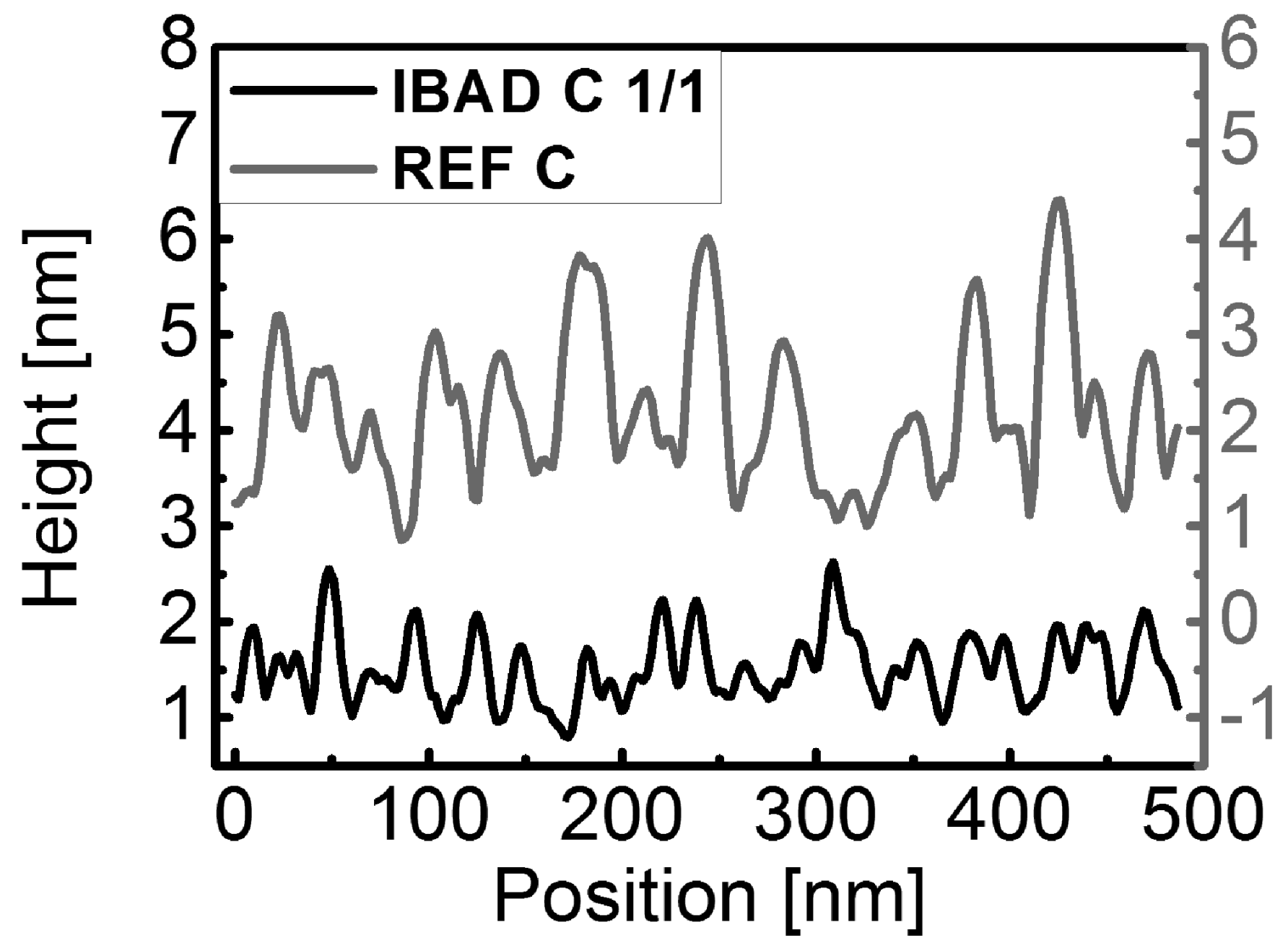

Figure 5 (Figure_5.tif) 


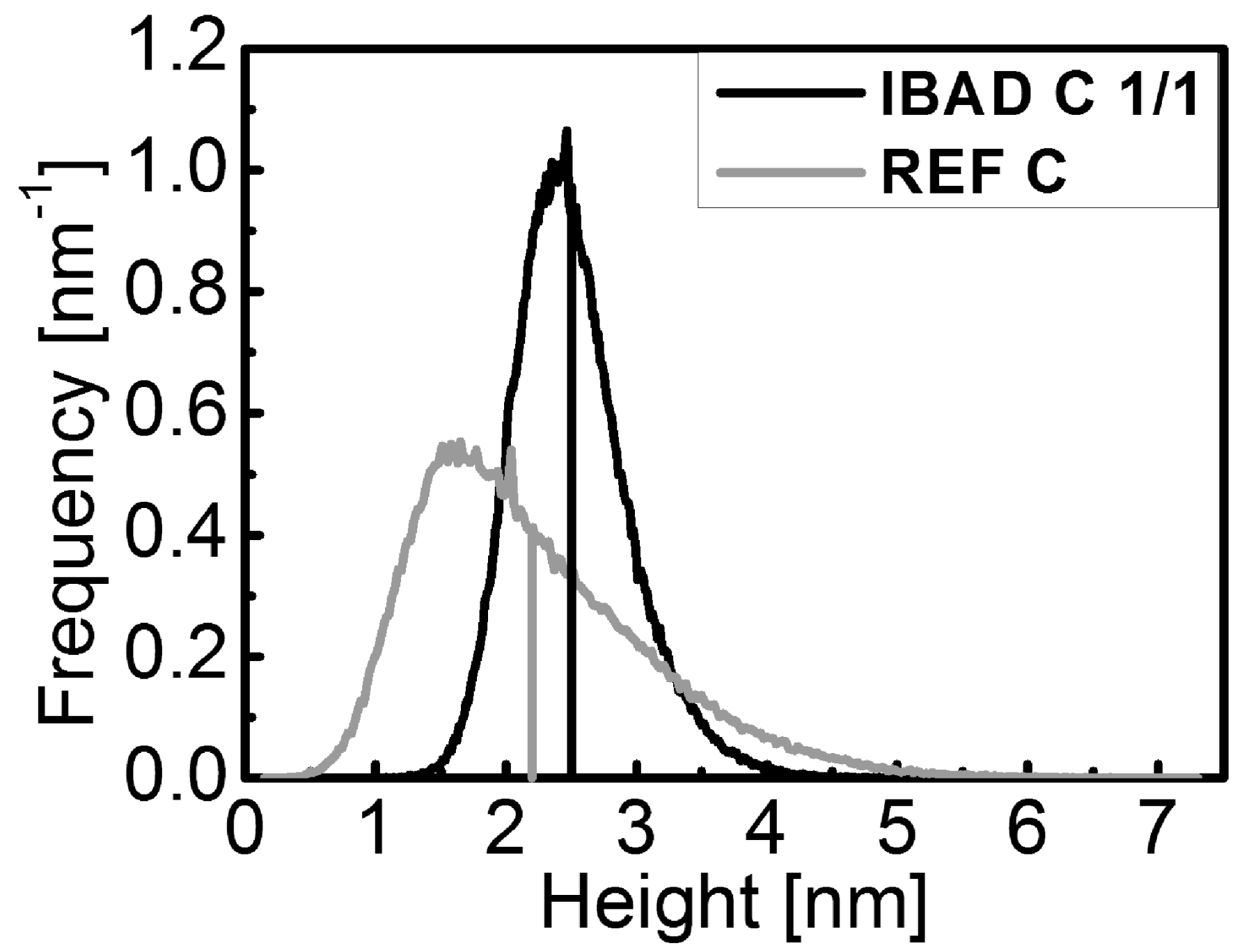

Figure 6 (Figure_6.tif) 


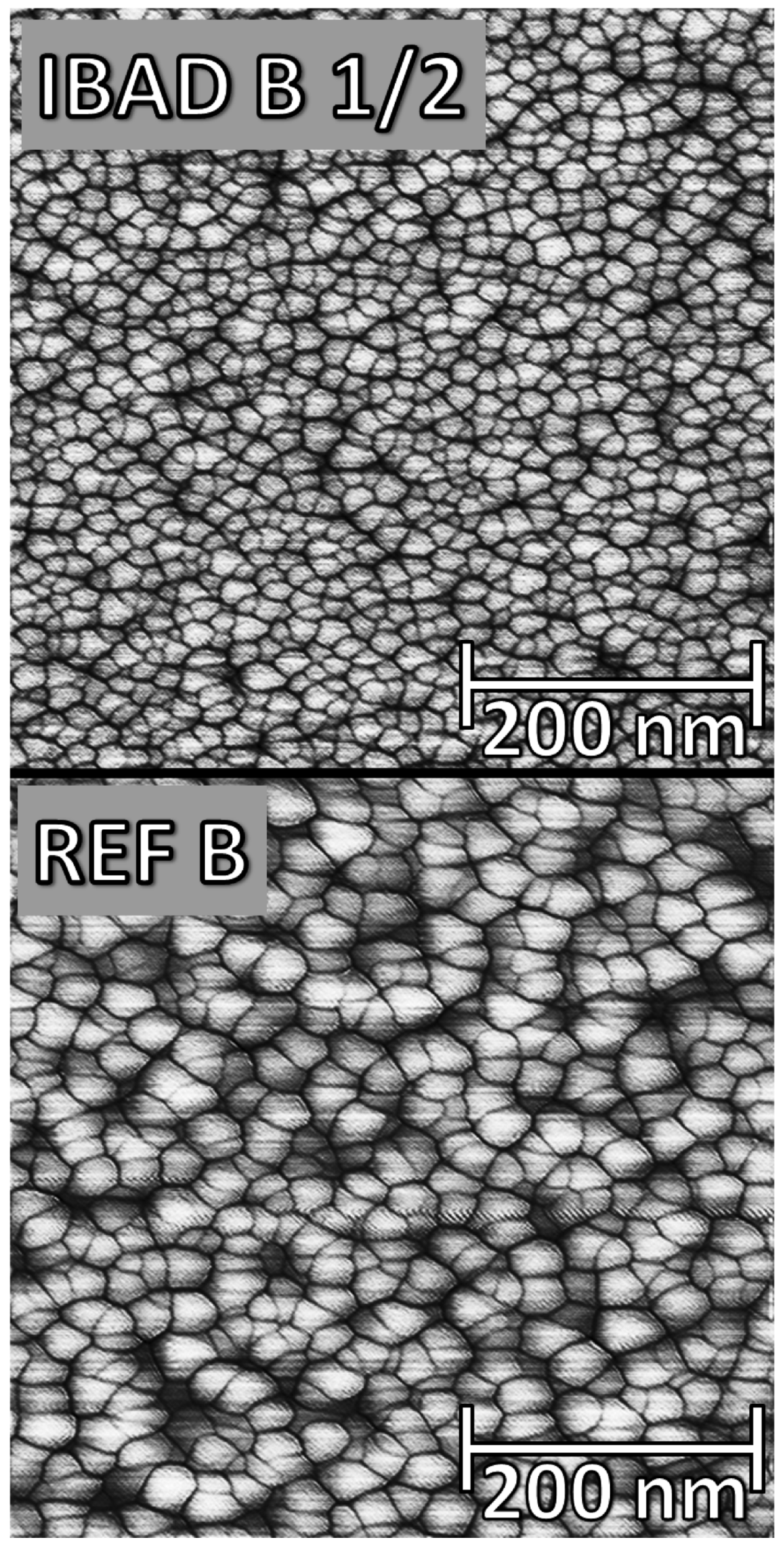

Figure 7 (Figure_7.tif) 


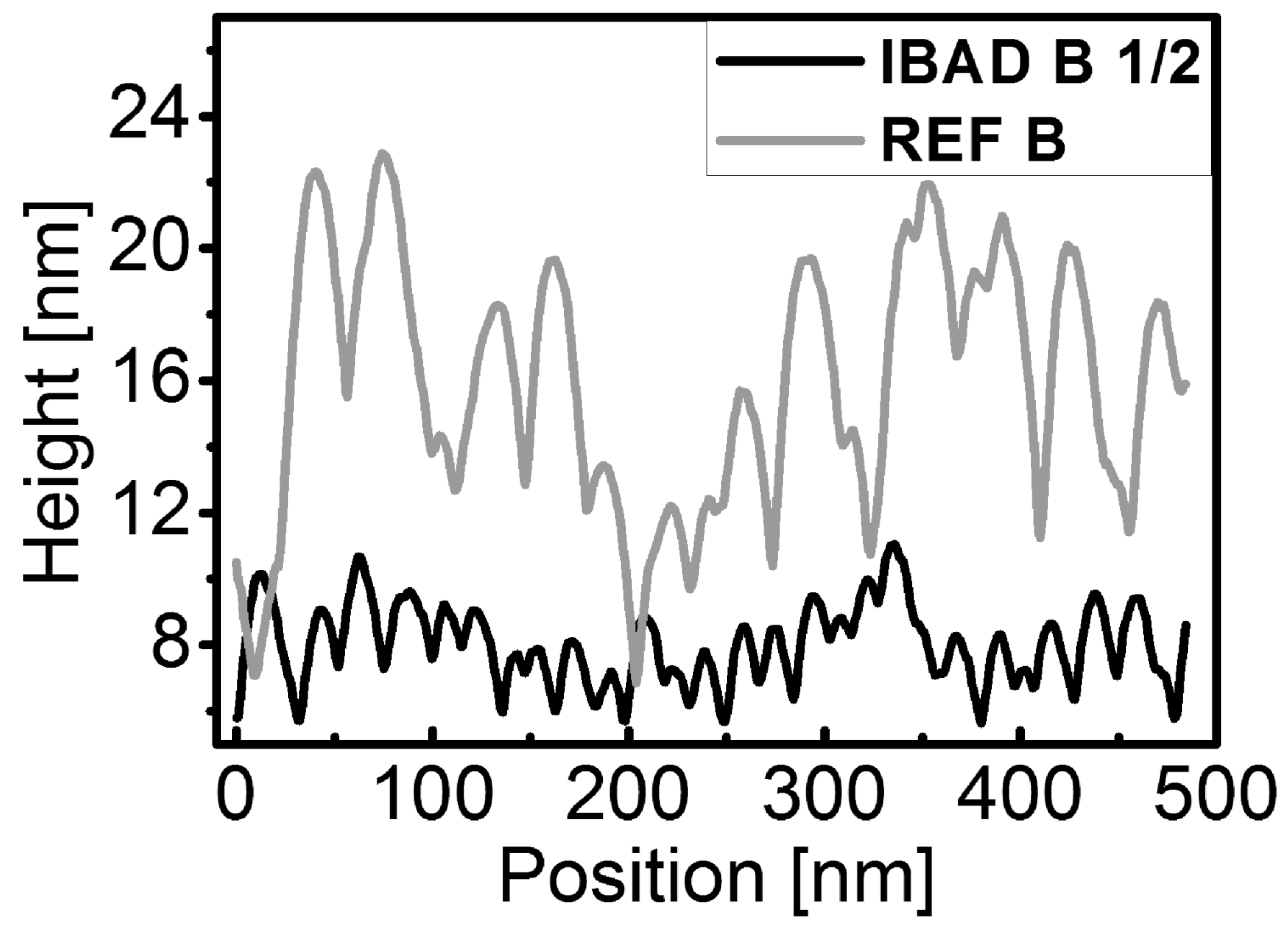

Figure 8 (Figure_8.tif) 


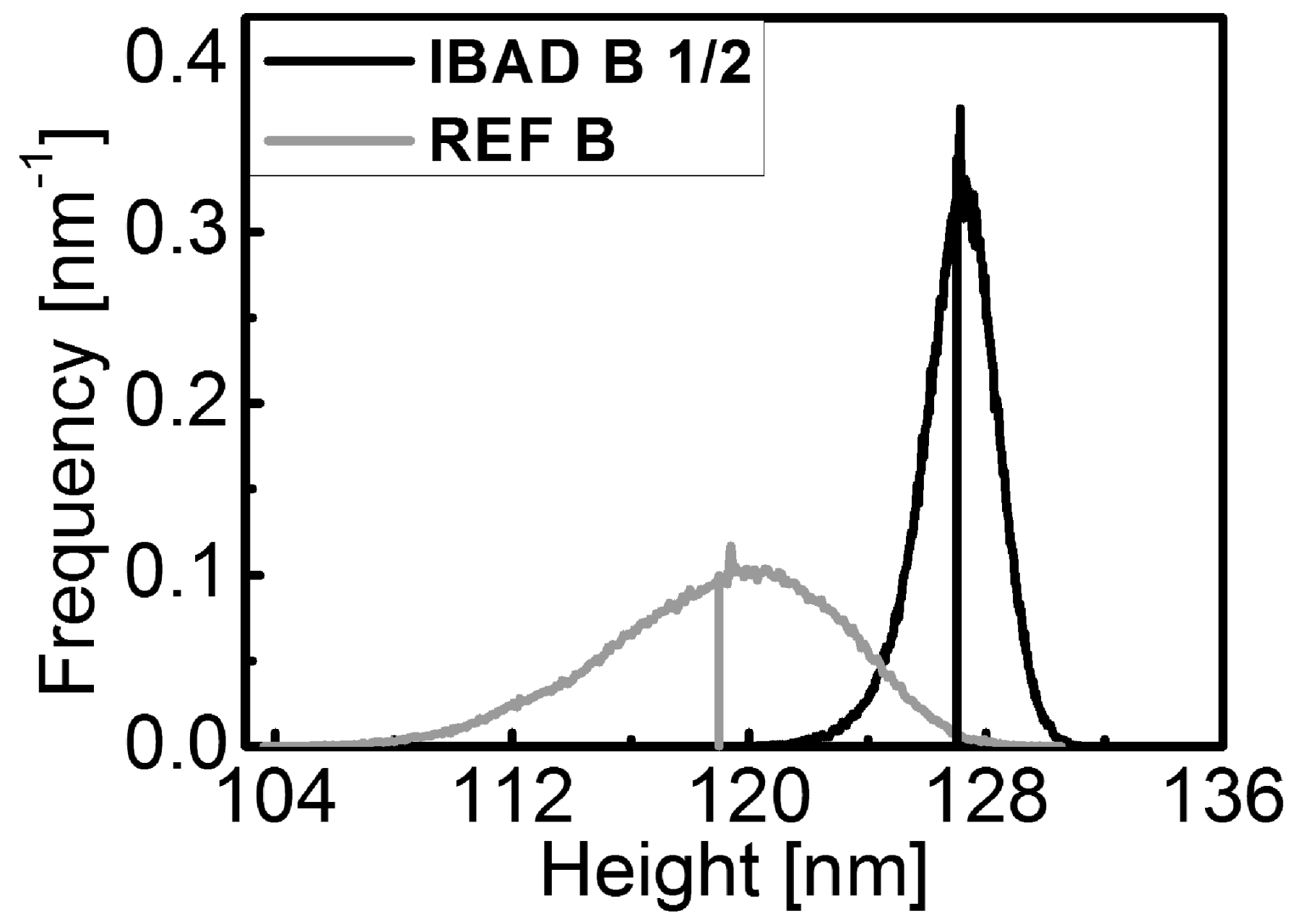

Figure 9 (Figure_9.tif) 


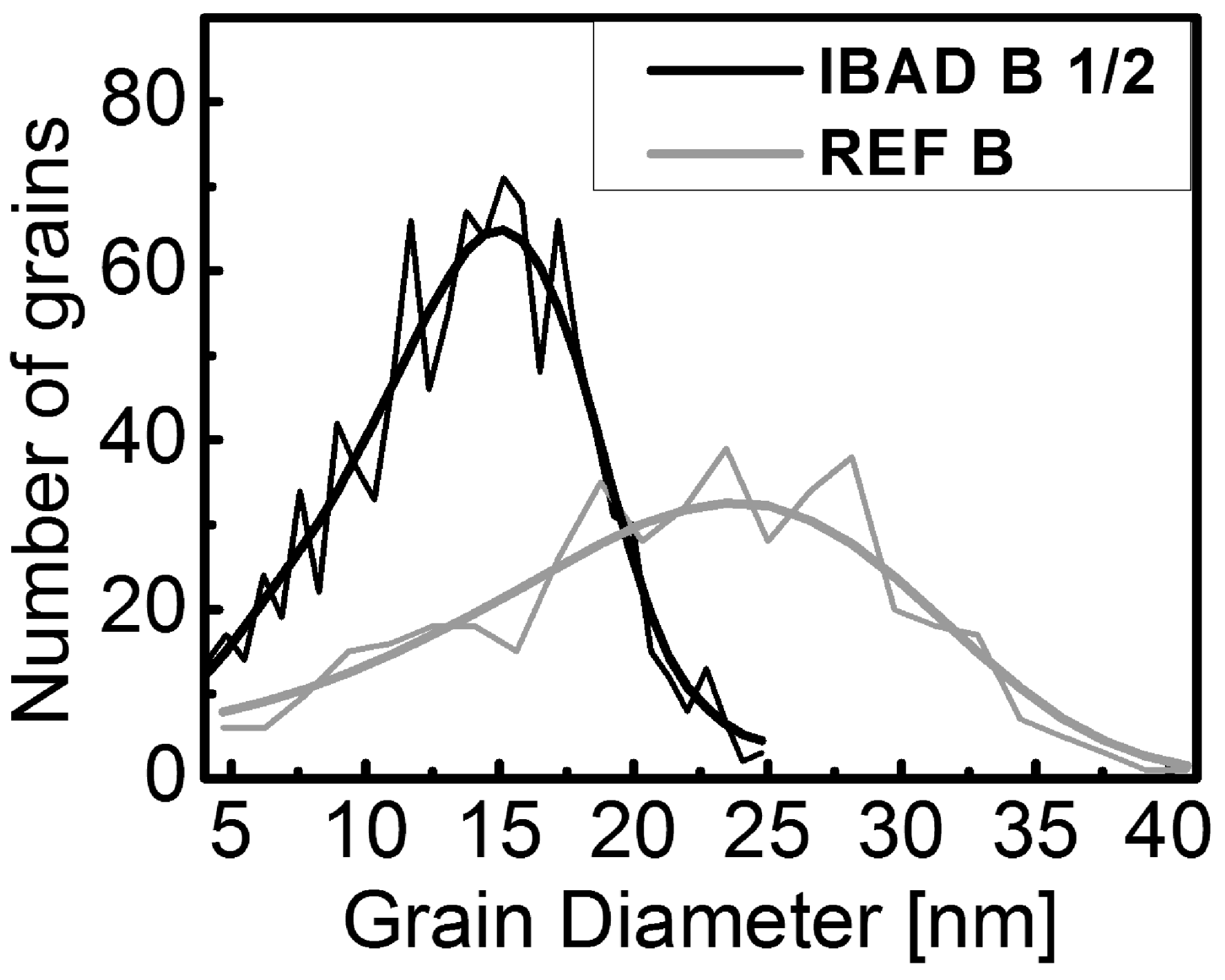

Figure 10 (Figure_10.tif) 


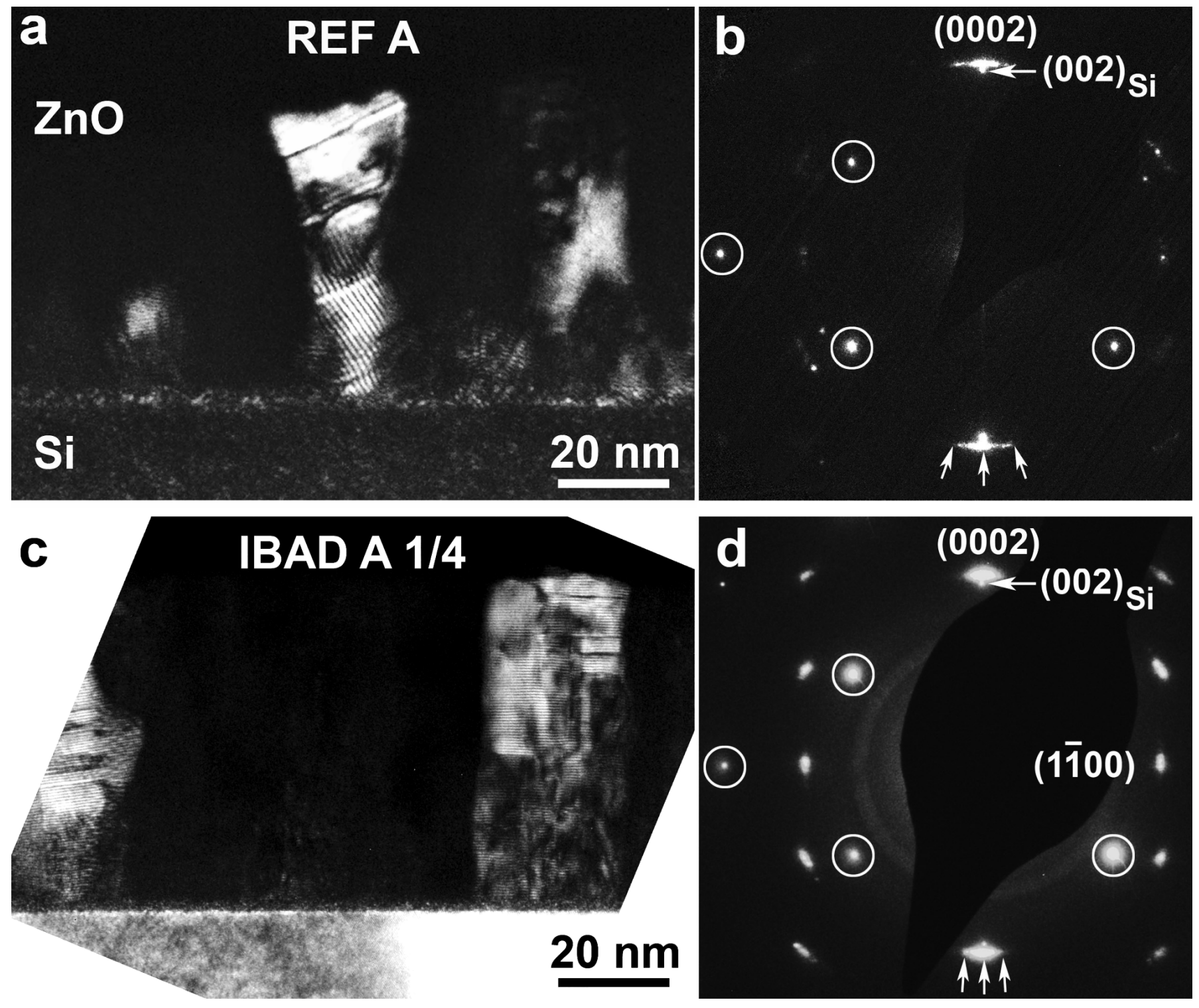

Figure 11 (Figure_11.tif) 


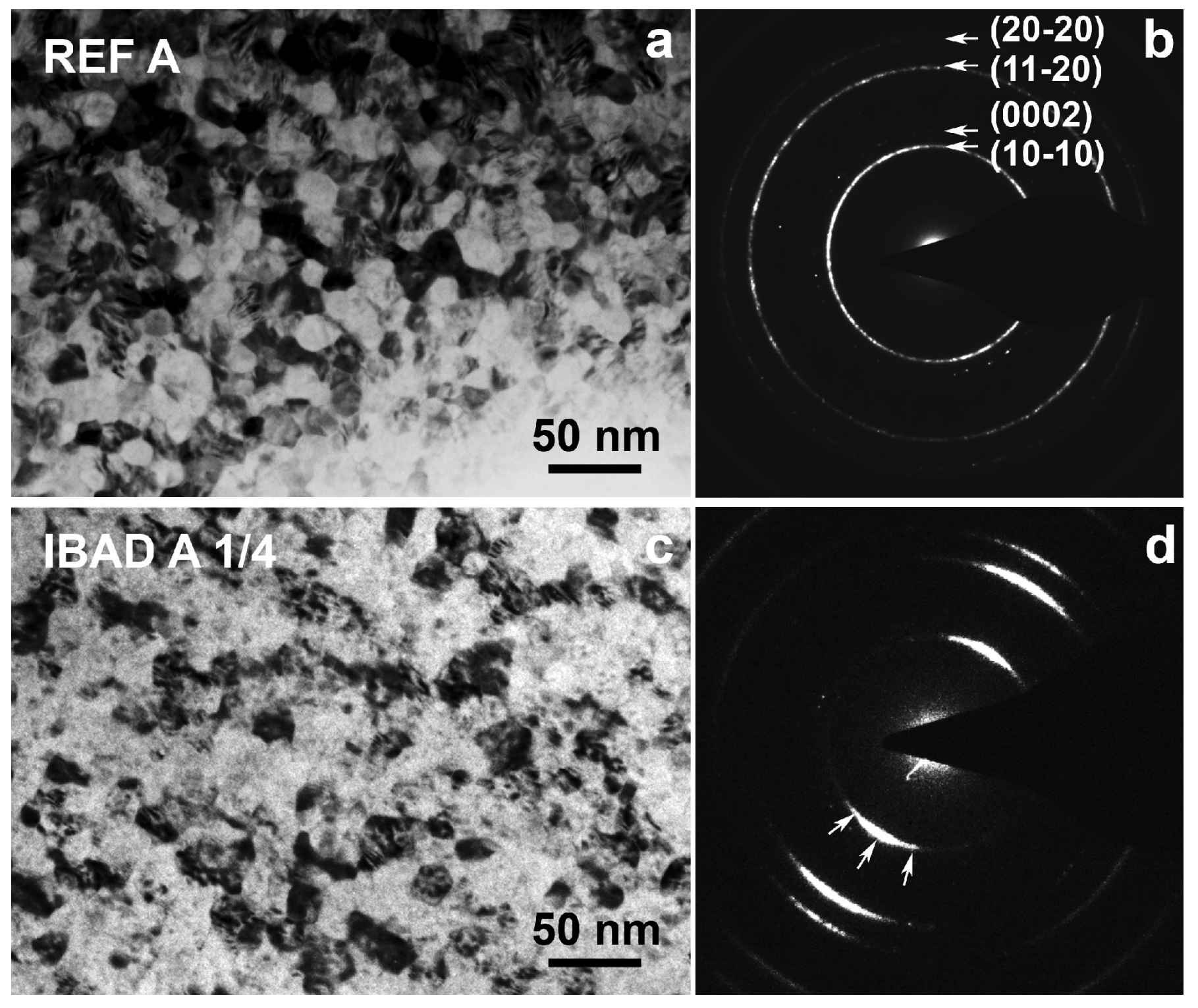

Figure 12 (Figure_12.tif) 


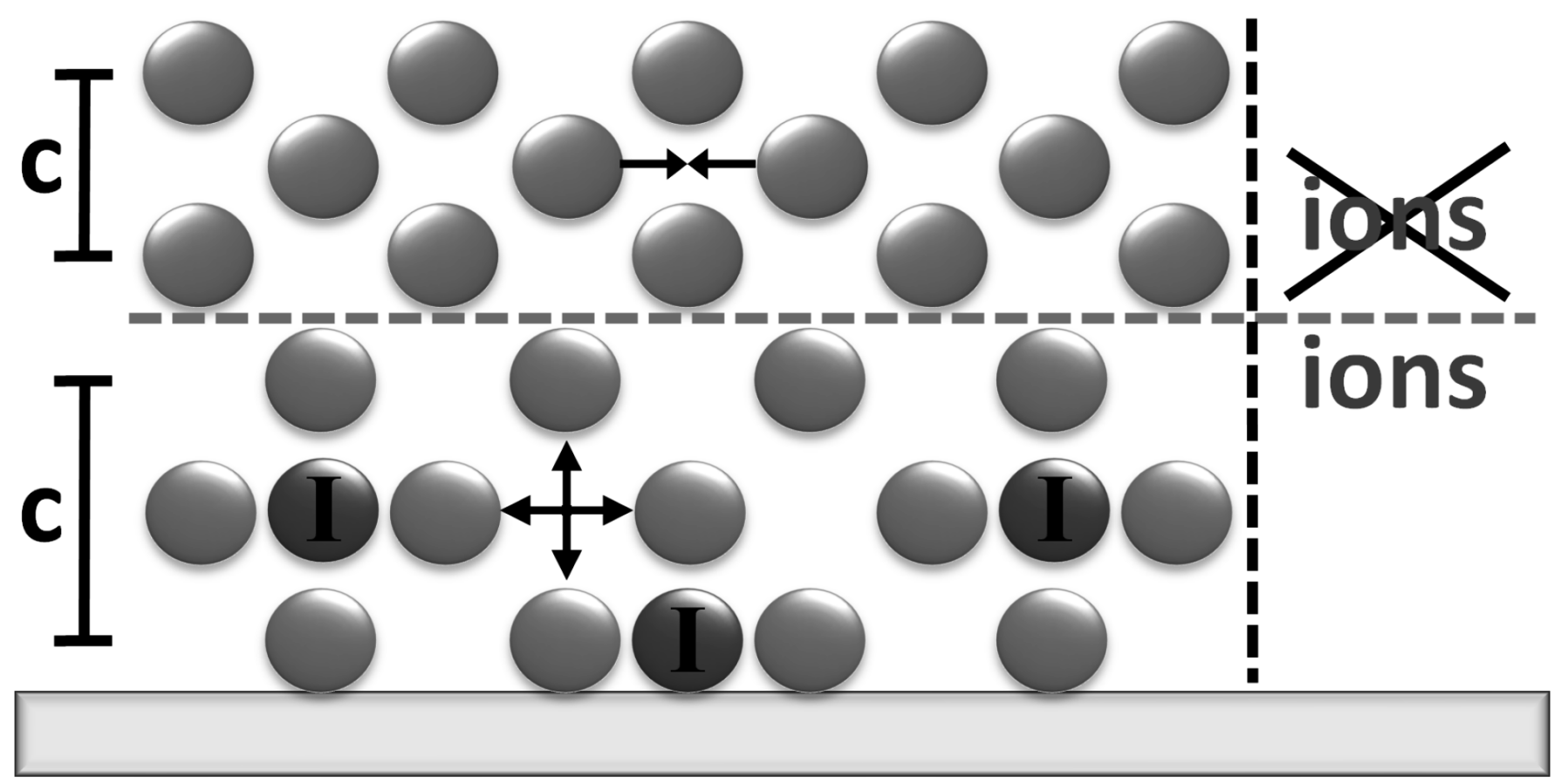

Figure 13 (Figure_13.tif) 


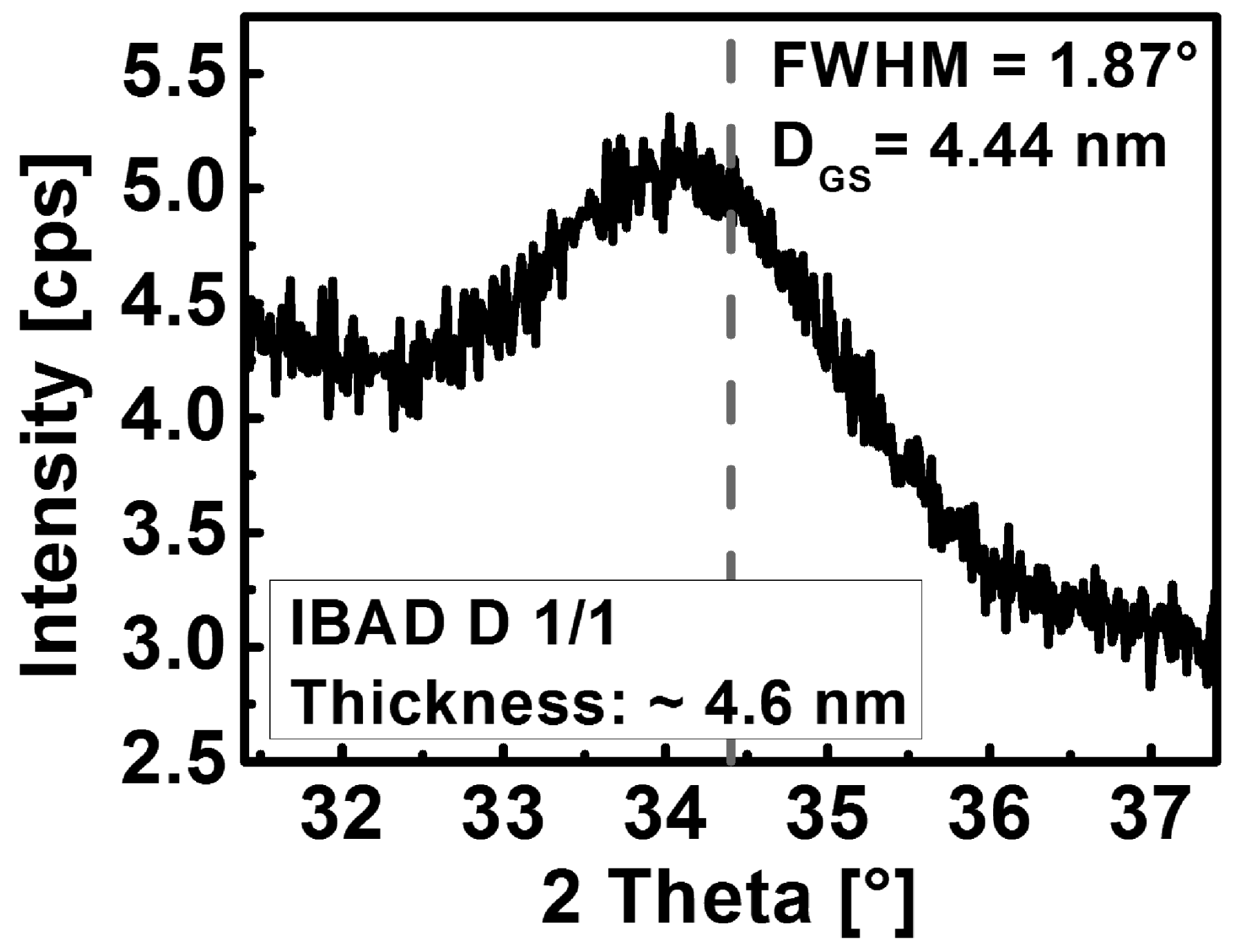

Figure 14 (Figure_14.tif) 


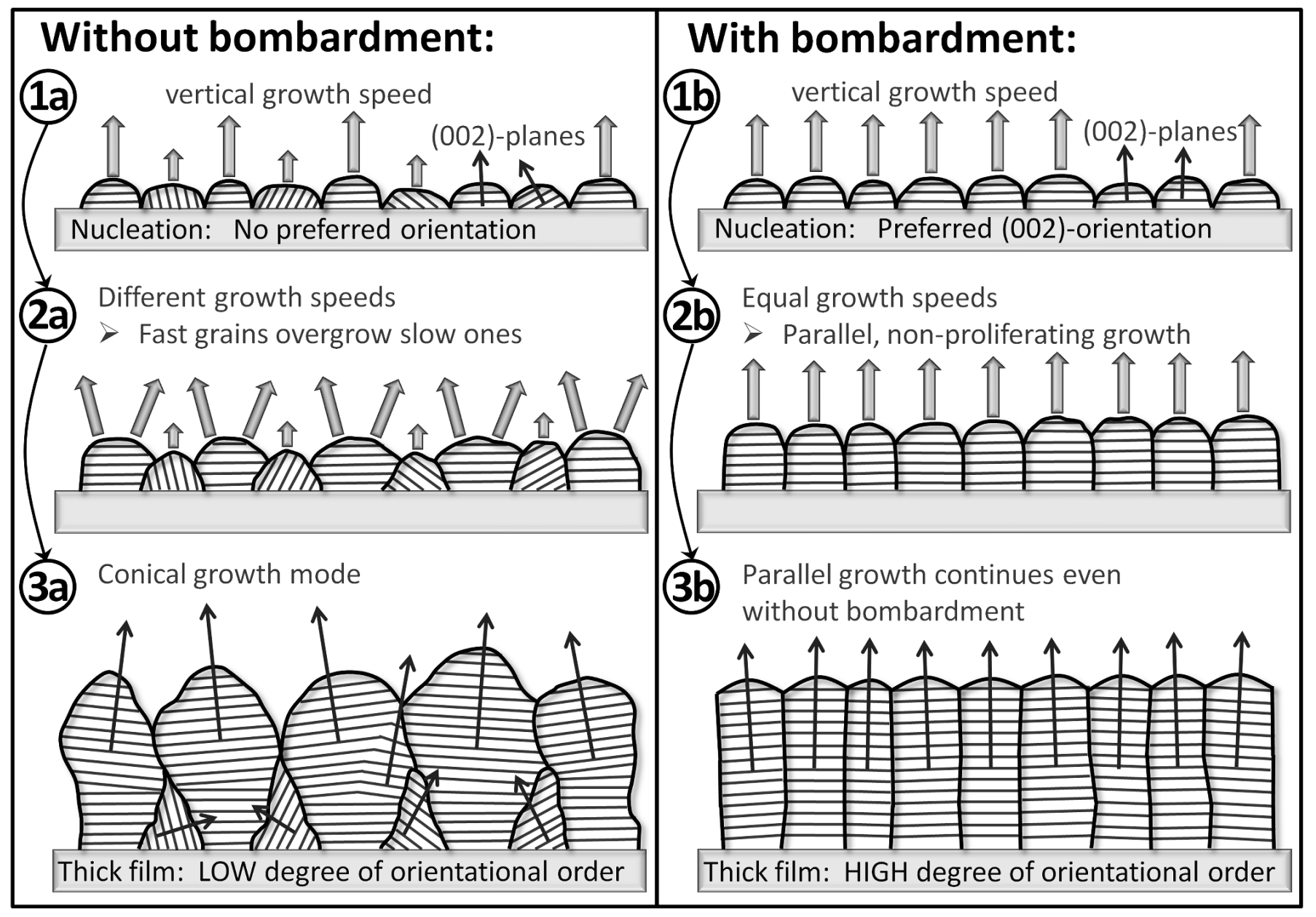

Figure 15 (Figure_15.tif) 